DESY 03-100

UAB-FT-551

CERN-TH/2003-199

\title{
Leptogenesis for Pedestrians
}

\author{
W. Buchmüller \\ Deutsches Elektronen-Synchrotron DESY, 22603 Hamburg, Germany \\ P. Di Bari \\ IFAE, Universitat Autònoma de Barcelona, \\ 08193 Bellaterra (Barcelona), Spain \\ M. Plümacher \\ Theory Division, CERN, 1211 Geneva 23, Switzerland
}

\begin{abstract}
During the process of thermal leptogenesis temperature decreases by about one order of magnitude while the baryon asymmetry is generated. We present an analytical description of this process so that the dependence on the neutrino mass parameters becomes transparent. In the case of maximal $C P$ asymmetry all decay and scattering rates in the plasma are determined by the mass $M_{1}$ of the decaying heavy Majorana neutrino, the effective light neutrino mass $\widetilde{m}_{1}$ and the absolute mass scale $\bar{m}$ of the light neutrinos. In the mass range suggested by neutrino oscillations, $m_{\text {sol }} \simeq 8 \times$ $10^{-3} \mathrm{eV} \lesssim \widetilde{m}_{1} \lesssim m_{\text {atm }} \simeq 5 \times 10^{-2} \mathrm{eV}$, leptogenesis is dominated just by decays and inverse decays. The effect of all other scattering processes lies within the theoretical uncertainty of present calculations. The final baryon asymmetry is dominantly produced at a temperature $T_{B}$ which can be about one order of magnitude below the heavy neutrino mass $M_{1}$. We also derive an analytical expression for the upper bound on the light neutrino masses implied by successful leptogenesis.
\end{abstract}




\section{Introduction and summary}

Leptogenesis [1] provides a simple and elegant explanation of the cosmological matterantimatter asymmetry. A beautiful aspect of this mechanism is the connection between the baryon asymmetry and neutrino properties. In its simplest version leptogenesis is dominated by the $C P$ violating interactions of the lightest of the heavy Majorana neutrinos, the seesaw partners of the ordinary neutrinos. The requirement of successful baryogenesis yields stringent constraints on the masses of light and heavy neutrinos. In particular, all light neutrino masses have to be smaller than $0.1 \mathrm{eV}[2]$.

Leptogenesis is closely related to classical GUT baryogenesis [3], where the deviation of the distribution function of some heavy particle from its equilibrium distribution provides the necessary departure from thermal equilibrium. The non-equilibrium process of baryogenesis is usually studied by means of Boltzmann equations [4, 5]. In the same way, leptogenesis has been studied during the past years, with increasing sophistication [6]-[12]. The goal of the present paper is to provide an analytical description of the leptogenesis process such that the dependence on the neutrino mass parameters becomes transparent. As we shall see this is important to understand the size of corrections to the simplest Boltzmann equations, which have to be taken into account to arrive eventually at a 'theory of leptogenesis'.

We will first consider the simplest case where the initial temperature $T_{\mathrm{i}}$ is larger than $M_{1}$, the mass of the lightest heavy neutrino $N_{1}$. We will also neglect decays of the two heavier neutrinos $N_{2}$ and $N_{3}$, assuming that a generation of $B-L$ asymmetry from their decays either does not occur at all or that it does not influence the final value of $B-L$. Further, we restrict ourselves to the non supersymmetric case, and we assume that the lightest heavy neutrino $N_{1}$ is the only relevant degree of freedom beyond the standard model particle species.

Within this minimal framework the Boltzmann equations can be written in the following form ${ }^{1}$,

$$
\begin{aligned}
\frac{d N_{N_{1}}}{d z} & =-(D+S)\left(N_{N_{1}}-N_{N_{1}}^{\mathrm{eq}}\right), \\
\frac{d N_{B-L}}{d z} & =-\varepsilon_{1} D\left(N_{N_{1}}-N_{N_{1}}^{\mathrm{eq}}\right)-W N_{B-L},
\end{aligned}
$$

where $z=M_{1} / T$. The number density $N_{N_{1}}$ and the amount of $B-L$ asymmetry, $N_{B-L}$, are calculated in a portion of comoving volume that contains one photon at temperatures $T \gg M_{1}$, so that the relativistic equilibrium $N_{1}$ number density is given by $N_{N_{1}}^{\text {eq }}(z \ll$

\footnotetext{
${ }^{1}$ We use the conventions of Ref. [10].
} 
1) $=3 / 4$. There are four classes of processes which contribute to the different terms in the equations: decays, inverse decays, $\Delta L=1$ scatterings and $\Delta L=2$ processes mediated by heavy neutrinos. The first three all modify the $N_{1}$ abundance and try to push it towards its equilibrium value $N_{N_{1}}^{\text {eq }}$. Denoting by $H$ the Hubble expansion rate, the term $D=\Gamma_{D} /(H z)$ accounts for decays and inverse decays, while the scattering term $S=\Gamma_{S} /(H z)$ represents the $\Delta L=1$ scatterings. Decays also yield the source term for the generation of the $B-L$ asymmetry, the first term in Eq. (2), while all other processes contribute to the total washout term $W=\Gamma_{W} /(H z)$ which competes with the decay source term. The expansion rate is given by

$$
H \simeq \sqrt{\frac{8 \pi^{3} g_{*}}{90}} \frac{M_{1}^{2}}{M_{\mathrm{Pl}}} \frac{1}{z^{2}} \simeq 1.66 g_{*} \frac{M_{1}^{2}}{M_{\mathrm{Pl}}} \frac{1}{z^{2}},
$$

where $g_{*}=g_{S M}=106.75$ is the total number of degrees of freedom, and $M_{\mathrm{Pl}}=1.22 \times$ $10^{19} \mathrm{GeV}$ is the Planck mass. Note that we have not included the $N_{1}$ degrees of freedom since, as we will see, in the preferred strong washout regime, the heavy neutrinos are non-relativistic when the baryon asymmetry is produced.

The two terms $D$ and $S$ depend on the effective neutrino mass [7], defined as

$$
\widetilde{m}_{1}=\frac{\left(m_{D}^{\dagger} m_{D}\right)_{11}}{M_{1}}
$$

which has to be compared with the equilibrium neutrino mass

$$
m_{*}=\frac{16 \pi^{5 / 2} \sqrt{g_{*}}}{3 \sqrt{5}} \frac{v^{2}}{M_{\mathrm{Pl}}} \simeq 1.08 \times 10^{-3} \mathrm{eV} .
$$

The decay parameter

$$
K=\frac{\Gamma_{D}(z=\infty)}{H(z=1)}=\frac{\widetilde{m}_{1}}{m_{*}}
$$

introduced in the context of ordinary GUT baryogenesis [3], controls whether or not $N_{1}$ decays are in equilibrium. Here $\Gamma_{D}(z=\infty) \equiv \widetilde{\Gamma}_{D}$ is the $N_{1}$ decay width. The washout term $W$ has two contributions, $W=W_{0}+\Delta W$; the first term only depends on $\widetilde{m}_{1}$, while the second one depends on the product $M_{1} \bar{m}^{2}$, where $\bar{m}^{2}=m_{1}^{2}+m_{2}^{2}+m_{3}^{2}$ is the sum of the light neutrino masses squared [10].

The solution for $N_{B-L}$ is the sum of two terms [3],

$$
N_{B-L}(z)=N_{B-L}^{\mathrm{i}} e^{-\int_{z_{\mathrm{i}}}^{z} d z^{\prime} W\left(z^{\prime}\right)}-\frac{3}{4} \varepsilon_{1} \kappa\left(z ; \widetilde{m}_{1}, M_{1} \bar{m}^{2}\right),
$$

where the second term describes $B-L$ production from $N_{1}$ decays. It is expressed in terms of the efficiency factor $\kappa[9]$ which does not depend on the CP asymmetry $\varepsilon_{1}$. In 
the following sections we shall use two integral expressions for the efficiency factor,

$$
\begin{aligned}
\kappa(z) & =\frac{4}{3} \int_{z_{\mathrm{i}}}^{z} d z^{\prime} D\left(N_{N_{1}}-N_{N_{1}}^{\mathrm{eq}}\right) e^{-\int_{z^{\prime}}^{z} d z^{\prime \prime} W\left(z^{\prime \prime}\right)} \\
& =-\frac{4}{3} \int_{z_{\mathrm{i}}}^{z} d z^{\prime} \frac{D}{D+S} \frac{d N_{N_{1}}}{d z^{\prime}} e^{-\int_{z^{\prime}}^{z} d z^{\prime \prime} W\left(z^{\prime \prime}\right)} .
\end{aligned}
$$

Here $N_{N_{1}}$ and $d N_{N_{1}} / d z^{\prime}$ are the solution of the first kinetic equation (1) and its derivative, respectively. The efficiency factor $\kappa(z)$ is normalized in such a way that its final value $\kappa_{\mathrm{f}}=\kappa(\infty)$ approaches one in the limit of thermal initial abundance of the heavy neutrinos $N_{1}$ and no washout $(W=0)$. In general, for $N_{N_{1}}^{\mathrm{i}} \leq N_{N_{1}}^{\mathrm{eq}}=3 / 4$, one has $\kappa_{\mathrm{f}} \leq 1$. The first term in Eq. (7) accounts for the possible generation of a $B-L$ asymmetry before $N_{1}$ decays, e.g. from decays of the two heavier neutrinos $N_{2}$ and $N_{3}$, or from a completely independent mechanism. In the following we shall neglect such an initial asymmetry $N_{B-L}^{\mathrm{i}}$. In [2] it was shown that for values $\widetilde{m}_{1}>m_{*}$ even large initial asymmetries are washed out for initial temperatures $T_{\mathrm{i}} \gtrsim M_{1}$.

The predicted baryon to photon number ratio has to be compared with the value $\eta_{B}$ measured at recombination. It is related to $N_{B-L}^{\mathrm{f}}=N_{B-L}(z=\infty)$ by $\eta_{B}=$ $\left(a_{\mathrm{sph}} / f\right) N_{B-L}^{\mathrm{f}}$. Here $a_{\mathrm{sph}}=28 / 79$ [13] is the fraction of $B-L$ asymmetry converted into a baryon asymmetry by sphaleron processes, and $f=N_{\gamma}^{\text {rec }} / N_{\gamma}^{\star}=2387 / 86$ is the dilution factor calculated assuming standard photon production from the onset of leptogenesis till recombination. Using Eq. (8), one then obtains

$$
\eta_{B}=\frac{3}{4} \frac{a_{\mathrm{sph}}}{f} \varepsilon_{1} \kappa_{\mathrm{f}} \equiv d \varepsilon_{1} \kappa_{\mathrm{f}} \simeq 0.96 \times 10^{-2} \varepsilon_{1} \kappa_{\mathrm{f}} .
$$

In the following sections we will study analytically the solutions of the kinetic equations, focusing in particular on the final value of the efficiency factor. We start in sect. 2 with the basic framework of decays and inverse decays. In the two regimes of weak $\left(\widetilde{m}_{1}<m_{*}\right)$ and strong $\left(\widetilde{m}_{1}>m_{*}\right)$ washout the efficiency factor is obtained analytically, which then leads to a simple interpolation valid for all values of $\widetilde{m}_{1} . \Delta L=1$ scatterings are added in sect. 3 , and the resulting lower bounds on the heavy neutrino mass $M_{1}$ and on the initial temperature $T_{\mathrm{i}}$ are discussed. In sect. 4 an analytic derivation of the upper bound on the light neutrino masses is given, and in sect. 5 various corrections are described which have to be taken into account in a theory of leptogenesis. In appendix A a detailed discussion of the $\Delta L=2$ processes in the resonance region is presented. In the case of maximal $C P$ violation the entire $\Delta L=2$ scattering cross section can be expressed in terms of $M_{1}, \widetilde{m}_{1}$ and $\bar{m}$. The resulting Boltzmann equations are compared with previously obtained results based on exact Kadanoff-Baym equations. In appendix $\mathrm{B}$ various useful formulae are collected. 
Recently, two potentially important, and usually neglected, effects on leptogenesis have been discussed: the $\Delta L=1$ processes involving gauge bosons $[11,12]$ and thermal corrections at high temperature [12]. Further, the strength of the $\Delta L=2$ washout term has been corrected [12] compared to previous analysis. However, the reaction densities for the gauge boson processes are presently controversial [11, 12]. Also the suggestion made in [12] to include thermal masses as kinematical masses in decay and scattering processes leads to an unconventional picture at temperatures $T>M_{1}$, which differs qualitatively from the situation at temperatures $T<M_{1}$. If thermal corrections are only included as propagator effects [14] their influence is small. This issue remains to be clarified. Fortunately, both effects are only important in the case of weak washout, i.e. for $\tilde{m}_{1}<m_{*}$, where the final baryon asymmetry is strongly dependent on initial conditions in any case. In the strong washout regime, $\widetilde{m}_{1}>m_{*}$, which appears to be favored by the present evidence for neutrino masses, they do not affect the final baryon asymmetry significantly. In the following we will therefore ignore gauge boson processes and thermal corrections. These questions will be addressed elsewhere.

The main results of this paper are summarized in the figures 6, 9 and 10. Fig. 6 illustrates that for the basic processes of decays and inverse decays the analytical approximation for the efficiency factor agrees well with the numerical result. The figure also demonstrates that scatterings lead to a departure from this basic picture only for values $K=\widetilde{m}_{1} / m_{*}<1$, where the final baryon asymmetry depends strongly on the initial conditions. Fig. 9 shows the dependence of the efficiency factor on initial conditions and on $\Delta L=1$ scatterings for different values of the effective Higgs mass $M_{h}$. Again, for $\widetilde{m}_{1}>m_{*}$ this dependence is small and, within the theoretical uncertainties, the efficiency factor is given by the simple power law

$$
\kappa_{f}=(2 \pm 1) \times 10^{-2}\left(\frac{0.01 \mathrm{eV}}{\widetilde{m}_{1}}\right)^{1.1 \pm 0.1}
$$

Knowing the efficiency factor, one obtains from Eqs. (10) and (118) the maximal baryon asymmetry. Fig. 10 shows the lower bound on the initial temperature $T_{\mathrm{i}}$ as function of $\widetilde{m}_{1}$. In the most interesting mass range favored by neutrino oscillations it is about one order of magnitude smaller than the lower bound on $M_{1}$. The smallest temperature $T_{\mathrm{i}}^{\text {min }} \simeq 3 \times 10^{9} \mathrm{GeV}$ is reached at $\widetilde{m}_{1} \simeq 2 \times 10^{-3} \mathrm{eV}$. In sect. 4 an analytic expression for the light neutrino mass bound is derived, which explicitly shows the dependence on the involved parameters. 


\section{Decays and inverse decays}

It is very instructive to consider first a simplified picture in which decays and inverse decays are the only processes. For consistency, also the real intermediate state contribution to the $2 \rightarrow 2$ processes has to be included. The kinetic equations (1) and (2) then reduce to

$$
\begin{aligned}
\frac{d N_{N_{1}}}{d z} & =-D\left(N_{N_{1}}-N_{N_{1}}^{\mathrm{eq}}\right) \\
\frac{d N_{B-L}}{d z} & =-\varepsilon_{1} D\left(N_{N_{1}}-N_{N_{1}}^{\mathrm{eq}}\right)-W_{I D} N_{B-L},
\end{aligned}
$$

where $W_{I D}$ is the contribution to the washout term due to inverse decays. From Eqs. (8) and (12) one obtains for the efficiency factor,

$$
\kappa(z)=-\frac{4}{3} \int_{z_{\mathrm{i}}}^{z} d z^{\prime} \frac{d N_{N_{1}}}{d z^{\prime}} e^{-\int_{z^{\prime}}^{z} d z^{\prime \prime} W_{I D}\left(z^{\prime \prime}\right)} .
$$

As we shall see, decays and inverse decays are sufficient to describe qualitatively many properties of the full problem.

After a discussion of several useful analytic approximations we will study in detail the two regimes of weak and strong washout. The insight into the dynamics of the nonequilibrium process gained from the investigation of these limiting cases will then allow us to obtain analytic interpolation formulae which describe rather accurately the entire parameter range. All results will be compared with numerical solutions of the kinetic equations.

\subsection{Analytic approximations}

Let us first recall some basic definitions and formulae. The decay rate takes the form [4],

$$
\Gamma_{D}(z)=\tilde{\Gamma}_{D}\left\langle\frac{1}{\gamma}\right\rangle
$$

where the thermally averaged dilation factor is given by the ratio of the modified Bessel functions $K_{1}$ and $K_{2}$,

$$
\left\langle\frac{1}{\gamma}\right\rangle=\frac{K_{1}(z)}{K_{2}(z)}
$$

and $\widetilde{\Gamma}_{D}$ is the decay width,

$$
\widetilde{\Gamma}_{D}=\frac{\widetilde{m}_{1} M_{1}^{2}}{8 \pi v^{2}}
$$




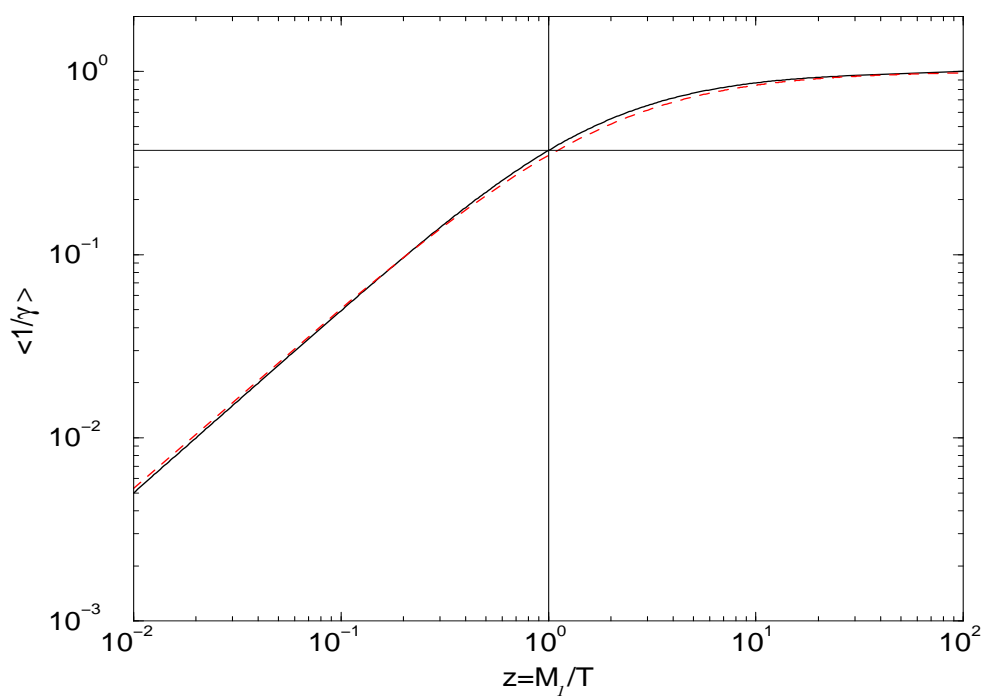

Figure 1: The dilation factor. The dashed line is the analytical expression Eq. (26) to be compared with the numerical result (solid line).

with the Higgs vacuum expectation value $v=174 \mathrm{GeV}$. The decay term $D$ is conveniently written in the form [3]

$$
D(z)=K z\left\langle\frac{1}{\gamma}\right\rangle .
$$

The inverse decay rate is related to the decay rate by

$$
\Gamma_{I D}(z)=\Gamma_{D}(z) \frac{N_{N_{1}}^{\mathrm{eq}}(z)}{N_{l}^{\mathrm{eq}}}
$$

where $N_{l}^{\mathrm{eq}}$ is the equilibrium density of lepton doublets. Since the number of degrees of freedom for heavy Majorana neutrinos and lepton doublets is the same, $g_{N_{1}}=g_{l}=2$, one has

$$
N_{N_{1}}^{\mathrm{eq}}(z)=\frac{3}{8} z^{2} K_{2}(z), \quad N_{l}^{\mathrm{eq}}=\frac{3}{4} .
$$

The contribution of inverse decays to the washout term $W$ is therefore

$$
W_{I D}(z)=\frac{1}{2} \frac{\Gamma_{I D}(z)}{H(z) z}=\frac{1}{4} K z^{3} K_{1}(z)
$$

which, together with Eqs. (16), (18) and (20), implies

$$
W_{I D}(z)=\frac{1}{2} D(z) \frac{N_{N_{1}}^{\mathrm{eq}}(z)}{N_{l}^{\mathrm{eq}}} .
$$


All relevant quantities are given in terms of the Bessel functions $K_{1}$ and $K_{2}$, whose asymptotic limits are well known. At high temperatures one has,

$$
K_{2}(z) \simeq \frac{2}{z} K_{1}(z) \simeq \frac{2}{z^{2}}, \quad z \ll 1,
$$

whereas at low temperatures,

$$
\begin{aligned}
K_{2}(z) & \simeq \frac{1}{z}\left(\frac{15}{8}+z\right) K_{1}(z) \\
& \simeq \frac{1}{z^{2}}\left(\frac{15}{8}+z\right) \sqrt{\frac{\pi}{2} z} e^{-z}, \quad z \gg 1
\end{aligned}
$$

Accurate interpolating functions for $K_{1}(z)$ and $K_{2}(z)$ for all values of $z$ are

$$
\begin{aligned}
K_{2}(z) & \simeq \frac{1}{z}\left(\frac{15}{8}+z\right) K_{1}(z) \\
& \simeq \frac{1}{z^{2}}\left(\frac{15}{8}+z\right) \sqrt{1+\frac{\pi}{2} z} e^{-z} .
\end{aligned}
$$

Note, that for $z \ll 1$ this approximation gives $K_{2}(z) \simeq 15 /\left(8 z^{2}\right)$ rather than the exact asymptotic form $2 / z^{2}$. However, the high temperature domain is not so important for baryogenesis and the approximation (25) is rather precise in the more relevant regime around $z \simeq 1$.

Eq. (25) yields very simple expressions for the dilation factor and the decay term,

$$
\left\langle\frac{1}{\gamma}\right\rangle(z) \simeq \frac{z}{\frac{15}{8}+z}, \quad D(z) \simeq K \frac{z^{2}}{\frac{15}{8}+z} .
$$

As Fig. 1 shows these analytical approximations are rather precise. The relative error is always less than 7\%. The washout term (21) becomes in the approximation (25),

$$
W_{I D}(z) \simeq \frac{1}{4} K z^{2} \sqrt{1+\frac{\pi}{2} z} e^{-z} .
$$

It is useful to define a value $z_{\mathrm{d}}$, corresponding to a 'decay temperature' $T_{\mathrm{d}}$ below which decays are in equilibrium, by $\Gamma_{D}\left(z_{\mathrm{d}}\right) / H\left(z_{\mathrm{d}}\right)=z_{\mathrm{d}} D\left(z_{\mathrm{d}}\right)=2$. The value of $z_{\mathrm{d}}$ is determined by $K$, and from Eq. (26) one obtains

$$
z_{\mathrm{d}}^{3}-\frac{2}{K}\left(z_{\mathrm{d}}+\frac{15}{8}\right) \simeq 0 .
$$

For $K \ll 1$, this yields $z_{\mathrm{d}} \simeq \sqrt{2 / K}$, whereas $z_{\mathrm{d}} \simeq(15 / 4 K)^{1 / 3}$ for $K \gg 1$. At $K \simeq 1$ one has $z_{\mathrm{d}} \simeq 2$.

Inverse decays are in equilibrium if $W_{I D}(z) \geq 1$. From Eq. (27) one easily finds that $W_{I D}(z)$ reaches its maximal value $W_{I D}\left(z_{\max }\right) \simeq 0.3 K$ at $z_{\max } \simeq 2.4$. Hence, for $K>3$, there exists an interval $z_{\text {in }} \leq z_{\max } \leq z_{\text {out }}$, where inverse decays are in equilibrium. For $K \lesssim 3$ no such interval exists and inverse decays are always out of equilibrium. 


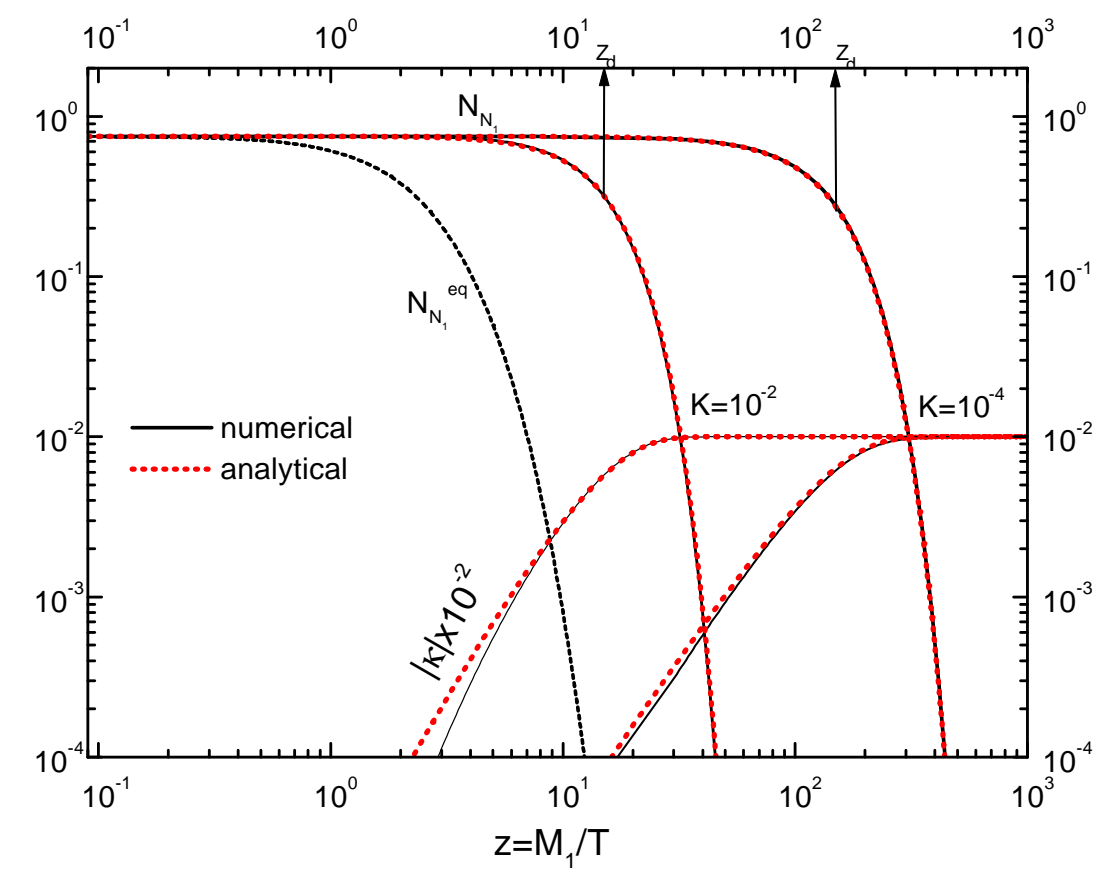

Figure 2: Out of equilibrium decays. $N_{1}$ number density, efficiency factor and decay temperature $T_{\mathrm{d}}=M_{1} / z_{\mathrm{d}}$ for $K=10^{-2}$ and $K=10^{-4}$.

\subsection{Out-of-equilibrium decays}

In the regime far out of equilibrium, $K \ll 1$, decays occur at very small temperatures, $z_{\mathrm{d}} \gg 1$, and the produced $B-L$ asymmetry is not reduced by washout effects. In this case the integral for the efficiency factor (14) becomes simply,

$$
\kappa(z) \simeq \frac{4}{3}\left(N_{N_{1}}^{\mathrm{i}}-N_{N_{1}}(z)\right)
$$

For $z<z_{\mathrm{d}}$ no asymmetry is generated because the heavy neutrinos do not decay. They also cannot be produced since inverse decays are switched off as well. Hence, in this regime the dynamics is completely frozen. For $z>z_{\mathrm{d}}$ the equilibrium abundance is negligible, and from Eq. (12) one finds,

$$
\begin{aligned}
N_{N_{1}}(z) & \simeq N_{N_{1}}^{\mathrm{i}} e^{-\int_{z_{\mathrm{i}}}^{z} d z^{\prime} D\left(z^{\prime}\right)} \\
& \simeq N_{N_{1}}^{\mathrm{i}} e^{-K\left(\frac{z^{2}}{2}-\frac{15 z}{8}+\left(\frac{15}{8}\right)^{2} \ln \left(1+\frac{8}{15} z\right)\right)} .
\end{aligned}
$$

Note that we have neglected the small neutrino abundance which for $N_{N_{1}}^{\mathrm{i}} \ll N_{N_{1}}^{\mathrm{eq}}$ is produced before the neutrinos decay. Fig. 2 shows the evolution of $N_{N_{1}}(z)$ and $N_{B-L}(z)$ 
for $K=10^{-2}$ and $K=10^{-4}$, with $N_{N_{1}}^{\mathrm{i}}=N_{N_{1}}^{\mathrm{eq}}=3 / 4$, comparing the numerical solutions with the analytical expressions.

The final value of the efficiency factor $\kappa_{\mathrm{f}}=\kappa(\infty)$ is proportional to the initial $N_{1}$ abundance. If $N_{1}^{\mathrm{i}}=N_{1}^{\mathrm{eq}}=3 / 4$, then $\kappa_{\mathrm{f}}=1$. But if the initial abundance is zero, then $\kappa_{\mathrm{f}}=0$ as well. Therefore in this region there is the well known problem that one has to invoke some external mechanism to produce the initial abundance of neutrinos. Moreover the assumption that the initial asymmetry is washed out does not hold. Thus in the regime $K \ll 1$ the results strongly depend on the initial conditions and the picture is not self-contained.

\subsection{Dynamical initial abundance}

In order to obtain the efficiency factor in the case of vanishing initial $N_{1}$-abundance, $N_{N_{1}}\left(z_{\mathrm{i}}\right) \equiv N_{N_{1}}^{\mathrm{i}} \simeq 0$, one has to calculate how heavy neutrinos are dynamically produced by inverse decays. This requires solving the kinetic equation (12) with the initial condition $N_{N_{1}}^{\mathrm{i}}=0$.

Let us define a value $z_{\text {eq }}$ by the condition

$$
N_{N_{1}}\left(z_{\mathrm{eq}}\right)=N_{N_{1}}^{\mathrm{eq}}\left(z_{\mathrm{eq}}\right)
$$

Eq. (12) implies that the number density reaches its maximum at $z=z_{\text {eq }}$. An approximate solution can be found by noting that for $z<z_{\text {eq }}$ inverse decays dominate and thus

$$
\frac{d N_{N_{1}}}{d z} \simeq D N_{N_{1}}^{\mathrm{eq}}>0
$$

A straightforward integration yields for $z<z_{\text {eq }}$ (cf. (16), (18), (20)),

$$
\begin{aligned}
N_{N_{1}}(z) & \simeq \frac{3}{8} K \int_{z_{\mathrm{i}}}^{z} d z^{\prime} z^{\prime 3} K_{1}\left(z^{\prime}\right) \\
& =\frac{3}{2} \int_{z_{\mathrm{i}}}^{z} d z^{\prime} W_{I D}\left(z^{\prime}\right) .
\end{aligned}
$$

In the case $z_{\mathrm{i}} \ll z<1$, this implies for the number density,

$$
N_{N_{1}}(z) \simeq \frac{K}{8} z^{3}
$$

where the small dependence on $z_{\mathrm{i}}$ has been neglected.

We can now calculate the corresponding approximate solution for the efficiency factor $\kappa(z)$. For $z<z_{\text {eq }}$ the efficiency factor $\kappa \equiv \kappa^{-}$is negative since, $N_{N_{1}}<N_{N_{1}}^{\text {eq }}$. From 
Eqs. (14) and (32) one obtains

$$
\begin{aligned}
\kappa^{-}(z) & \simeq-\frac{4}{3} \int_{z_{\mathrm{i}}}^{z} d z^{\prime} D\left(z^{\prime}\right) N_{N_{1}}^{\mathrm{eq}}\left(z^{\prime}\right) e^{-\int_{z^{\prime}}^{z} d z^{\prime \prime} W_{I D}\left(z^{\prime \prime}\right)} \\
& =-2 \int_{z_{\mathrm{i}}}^{z} d z^{\prime} W_{I D}\left(z^{\prime}\right) e^{-\int_{z^{\prime}}^{z} d z^{\prime \prime} W_{I D}\left(z^{\prime \prime}\right)} \\
& =-2\left(1-e^{-\int_{z_{\mathrm{i}}}^{z} d z^{\prime} W_{I D}\left(z^{\prime}\right)}\right) \\
& \simeq-2\left(1-e^{-\frac{2}{3} N_{N_{1}}(z)}\right), \quad z \leq z_{\mathrm{eq}} .
\end{aligned}
$$

As expected, for $N_{N_{1}} \ll 1$ the efficiency factor is proportional to $N_{N_{1}}$, up to corrections which correspond to washout effects. For $z>z_{\mathrm{eq}}, \kappa^{-}(z)$ is reduced by washout effects.

For $z \geq z_{\text {eq }}$, there is an additional positive contribution to the efficiency factor,

$$
\kappa^{+}(z) \simeq \frac{4}{3} \int_{z_{\mathrm{eq}}}^{z} d z^{\prime} D\left(z^{\prime}\right)\left(N_{N_{1}}\left(z^{\prime}\right)-N_{N_{1}}^{\mathrm{eq}}\left(z^{\prime}\right)\right) e^{-\int_{z^{\prime}}^{z} z z^{\prime \prime} W_{I D}\left(z^{\prime \prime}\right)} .
$$

The total efficiency factor is the sum of both contributions,

$$
\kappa_{\mathrm{f}}(z)=\kappa^{+}(z)+\kappa^{-}(z) .
$$

For $z \geq z_{\text {eq }}$ we now have to distinguish two different situations, the weak and strong washout regimes, respectively.

\subsubsection{Weak washout regime}

Consider first the case of weak washout, $K \ll 1$, which implies $z_{\text {eq }} \gg 1$. From Eq. (33) one then finds,

$$
N_{N_{1}}\left(z_{\text {eq }}\right) \simeq \frac{9 \pi}{16} K \equiv N(K) .
$$

A solution for $N_{N_{1}}(z)$, valid for any $z$, is obtained by using in Eq. (33) the useful approximation

$$
\int_{0}^{z} d z^{\prime} z^{\prime 3} K_{1}\left(z^{\prime}\right) \simeq \frac{3 \pi z^{3}}{\left[(9 \pi)^{c}+\left(2 z^{3}\right)^{c}\right]^{1 / c}},
$$

with $c=0.7$. This yields an interpolation of the two asymptotic regimes (cf. Eqs. (34) and (38)), which is in excellent agreement with the numerical result, as shown in Fig. 3a for $K=10^{-2}$.

For $z>z_{\mathrm{eq}} \gg 1$ decays dominate over inverse decays, such that

$$
\frac{d N_{N_{1}}}{d z}=-D N_{N_{1}}<0 \text {. }
$$


In this way one easily obtains

$$
N_{N_{1}}(z)=N_{N_{1}}^{\mathrm{eq}}\left(z_{\mathrm{eq}}\right) e^{-\int_{z \mathrm{eq}}^{z} d z^{\prime} D\left(z^{\prime}\right)} .
$$

Moreover, for $z>z^{\mathrm{eq}}, W_{I D}(z)$ is exponentially suppressed and washout effects can be neglected in first approximation. For the negative part of the efficiency factor one then has (cf. Eq. (35))

$$
\kappa^{-}(z)=-2\left(1-e^{-\frac{2}{3} N(K)}\right)
$$

From Eq. (29) one obtains for the positive contribution,

$$
\kappa^{+}(z)=\frac{4}{3}\left(N(K)-N_{N_{1}}(z)\right) .
$$

The final efficiency factor is then given by

$$
\kappa_{\mathrm{f}}(K) \simeq \frac{4}{3} N(K)-2\left(1-e^{-\frac{2}{3} N(K)}\right)
$$

To first order in $N(K) \propto K$ the final efficiency factor vanishes. This corresponds to the approximation where washout effects are completely neglected. As discussed above, $\kappa_{\mathrm{f}}$ is then proportional to $N_{N_{1}}^{\mathrm{i}}$ and therefore zero. To obtain a non-zero asymmetry the washout in the period $z<z_{\text {eq }}$ is crucial. It reduces the absolute value of the negative contribution $\kappa^{-}(z)$, yielding a positive efficiency factor of order $\mathcal{O}\left(K^{2}\right)$,

$$
\kappa_{\mathrm{f}}(K)=\left[\frac{2}{3} N(K)\right]^{2} \simeq \frac{9 \pi^{2}}{64} K^{2} .
$$

Such a reduction of the generated asymmetry has previously been observed in the context of GUT baryogenesis [15]. Note, that for $K>1$ Eq. (45) does not hold, since in this case $z_{\text {eq }}$ becomes small and washout effects for $z \geq z_{\text {eq }}$ are also important.

In Fig. 3a the analytical solutions for $N_{N_{1}}(z)$ and $\left|N_{B-L}(z)\right|=(3 / 4)\left|\varepsilon_{1} \kappa(z)\right|$ are compared with the numerical results for $K \simeq 10^{-2}$. A residual asymmetry survives after $z_{\mathrm{d}} \gg z_{\text {eq }}$ as remnant of the cancellation between the negative and the positive contributions to the efficiency factor. The second one is prevalent because washout suppresses $\kappa^{-}$more efficiently. As one can see in Fig. 3a, the analytical solution for the asymmetry slightly underestimates the final numerical value. This is because for $K \gtrsim 10^{-2}$ the approximation of neglecting washout for $z \geq z_{\mathrm{eq}}$ becomes inaccurate.

\subsubsection{Strong washout regime}

As $K$ increases, $z_{\text {eq }}$ decreases, and at $K \simeq 3$ the maximal number density $N(K)$ reaches the equilibrium density $N_{e q}$ at $z_{\text {eq }} \simeq 1$. For $K \gg 1$, one obtains from Eq. (34), $z_{\text {eq }} \simeq$ 

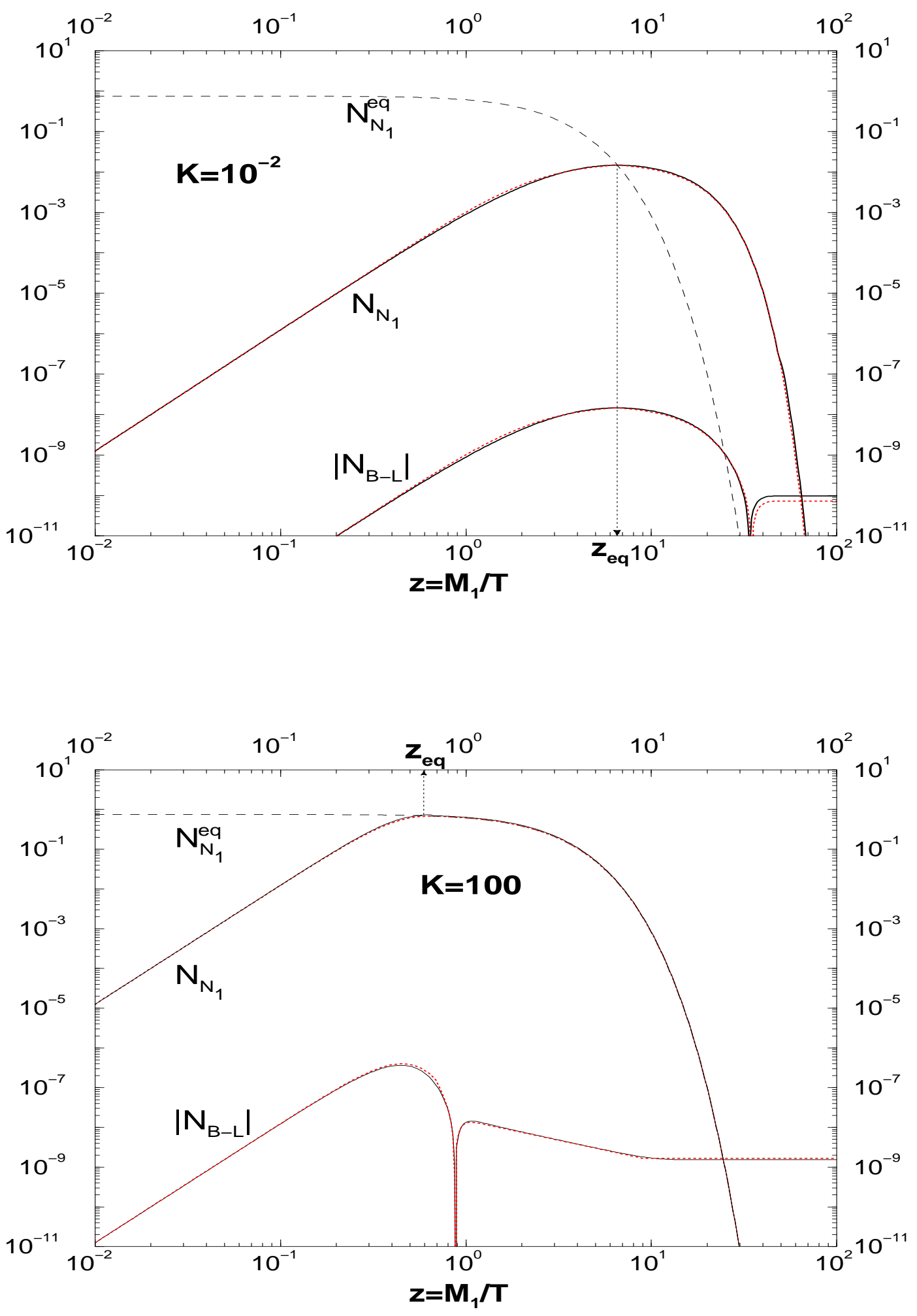

Figure 3: Comparison analytical (dashed lines) and numerical (solid lines) results for heavy neutrino production and $B-L$ asymmetry in the case of zero initial abundance, $N_{N_{1}}^{\mathrm{i}}=0$, for weak washout (top) and strong washout (bottom); $\left|\varepsilon_{1}\right|=10^{-6}$. 
$(6 / K)^{1 / 3} \simeq z_{\mathrm{d}} \ll 1$. A more accurate description for $z \lesssim z_{\text {eq }}$ has to take into account decays in addition to inverse decays, i.e. one has to solve Eq. (12). Since $z_{\text {eq }} \ll 1$, one can use $N_{N_{1}}^{\mathrm{eq}} \simeq 3 / 4$, and one then easily finds,

$$
N_{N_{1}}(z)=\frac{3}{4}\left(1-e^{-\frac{1}{6} K z^{3}}\right),
$$

which correctly reproduces Eq. (34) for $z \ll z_{\text {eq. }}$. An example, with $K=100$, is shown in Fig. $3 \mathrm{~b}$ which illustrates how well the analytical expression for neutrino production agrees with the numerical result.

Consider now the efficiency factor. For $K \gg 1$ we can neglect the negative contribution $\kappa^{-}$, assuming that the asymmetry generated at high temperatures is efficiently washed out. This is practically equivalent to assuming thermal initial abundance. We will see in the next section how to describe the transition from the weak to the strong washout regime.

For $K \gtrsim 3$, inverse decays are in equilibrium in the range $z_{\text {in }}<z<z_{\text {out }}$, with $z_{\text {in }} \simeq 2 / \sqrt{K}$. In the strong washout regime, $K \gg 1$, the efficiency factor can again be calculated analytically.

For $z \lesssim z_{\mathrm{d}} \simeq[15 /(4 K)]^{1 / 3}$ decays are not effective in tracking the equilibrium distribution. For the difference

$$
\Delta=N_{N_{1}}(z)-N_{N_{1}}^{\mathrm{eq}}(z),
$$

with $N_{N_{1}}(0)=N_{N_{1}}^{\mathrm{eq}}(0)=3 / 4 \equiv N_{\mathrm{eq}}$, one has,

$$
\Delta(z) \simeq N_{N_{1}}^{\mathrm{i}}-N_{N_{1}}^{\mathrm{eq}}(z) \simeq \frac{3}{16} z^{2}, \quad z \lesssim z_{\mathrm{d}} .
$$

The corresponding efficiency factor is given by (cf. (8))

$$
\kappa(z) \simeq \frac{4}{3} \int_{z_{\mathrm{i}}}^{z} d z^{\prime} D\left(z^{\prime}\right) \Delta\left(z^{\prime}\right) \simeq \frac{2 K}{75} z^{5}, \quad z \lesssim z_{\mathrm{d}} .
$$

On the other hand, for $z>z_{\mathrm{d}}$ the neutrino abundance tracks closely the equilibrium behavior. Since $D \propto K$, one can solve Eq. (12) systematically in powers of $1 / K$, which yields

$$
\Delta(z)=-\frac{1}{D} \frac{d N_{N_{1}}^{\mathrm{eq}}}{d z}+\mathcal{O}\left(\frac{1}{K^{2}}\right) .
$$

Using the properties of Bessel functions, Eq. (20) yields for the derivative of the equilibrium density,

$$
\frac{d N_{N_{1}}^{\mathrm{eq}}}{d z}=-\frac{3}{8} z^{2} K_{1}(z)=-\frac{3}{2 K z} W_{I D}(z) .
$$


We can now calculate the efficiency factor. From Eqs. (14) and (51) one obtains

$$
\begin{aligned}
\kappa(z) & =\frac{2}{K} \int_{z_{\mathrm{i}}}^{z} d z^{\prime} \frac{1}{z^{\prime}} W_{I D}\left(z^{\prime}\right) e^{-\int_{z^{\prime}}^{z} d z^{\prime \prime} W_{I D}\left(z^{\prime \prime}\right)} \\
& \equiv \int_{z_{\mathrm{i}}}^{z} d z^{\prime} e^{-\psi\left(z^{\prime}, z\right)} .
\end{aligned}
$$

The integral is dominated by the contribution from a region around the value $\bar{z}$ where $\psi\left(z^{\prime}, z\right)$ has a minimum. The condition for a local minimum $z_{\mathrm{B}}$, the vanishing of the first derivative, yields

$$
W_{I D}\left(z_{\mathrm{B}}\right)=\left\langle\frac{1}{\gamma}\right\rangle^{-1}\left(z_{\mathrm{B}}\right)-\frac{3}{z_{\mathrm{B}}} .
$$

Since the second derivative at $z_{\mathrm{B}}$ is positive one has $\bar{z}=\min \left\{z, z_{\mathrm{B}}\right\}$.

The integral (52) can be evaluated systematically by the steepest descent method (cf. [3]). Alternatively, a simple and very useful approximate analytical solution can be obtained by replacing in the exponent of the integrand $W_{I D}(z)$ by

$$
\bar{W}_{I D}(z)=\frac{\bar{z}}{z} W_{I D}(z)=-\frac{K \bar{z}}{4} \frac{d}{d z}\left(z^{2} K_{2}(z)\right) .
$$

The efficiency factor then becomes

$$
\begin{aligned}
\kappa(z) & \simeq \frac{2}{K \bar{z}} \int_{z_{\mathrm{i}}}^{z} d z^{\prime} \bar{W}_{I D}\left(z^{\prime}\right) e^{-\int_{z^{\prime}}^{z} d z^{\prime \prime} \bar{W}_{I D}\left(z^{\prime \prime}\right)} \\
& =\frac{2}{K \bar{z}}\left(1-e^{\left.-\int_{z_{\mathrm{i}}}^{z} z^{\prime} \bar{W}_{I D}\left(z^{\prime}\right)\right)}\right) .
\end{aligned}
$$

It is now easy to understand the behavior of $\kappa(z)$. For $z_{\mathrm{d}} \lesssim z<z_{\mathrm{B}}$, one has $\kappa \propto 1 / z$, while for $z \geq z_{\mathrm{B}}$ the efficiency factor gets frozen at a final value $\kappa_{\mathrm{f}} \simeq 2 /\left(K z_{\mathrm{B}}\right)$, up to a small correction $\mathcal{O}(\exp (-K))$.

One can also easily find global solutions for all values of $z$ by interpolating the asymptotic solutions for $z<z_{\mathrm{d}}$ and $z>z_{\mathrm{d}}$, respectively. From Eqs. (18), (48), (50) and (51) one obtains for the difference between $N_{1}$-abundance and equilibrium abundance,

$$
\Delta(z) \simeq\left(1+\frac{K z^{3}}{\frac{15}{4}+2 z}\right)^{-1} \frac{3}{16} z^{3} K_{1}(z) .
$$

Similarly, an interpolation between the expressions (49) and (55) for the efficiency factor is given by

$$
\kappa(z) \simeq\left(1+\frac{K^{2} \bar{z} z^{5}}{75}\right)^{-1} \frac{2 K}{75} z^{5} .
$$

A typical example of strong washout is shown in Fig. 4 for the value $K=100$, as in Fig. 3b, but now for thermal initial abundance. In this figure we also show the decay, 


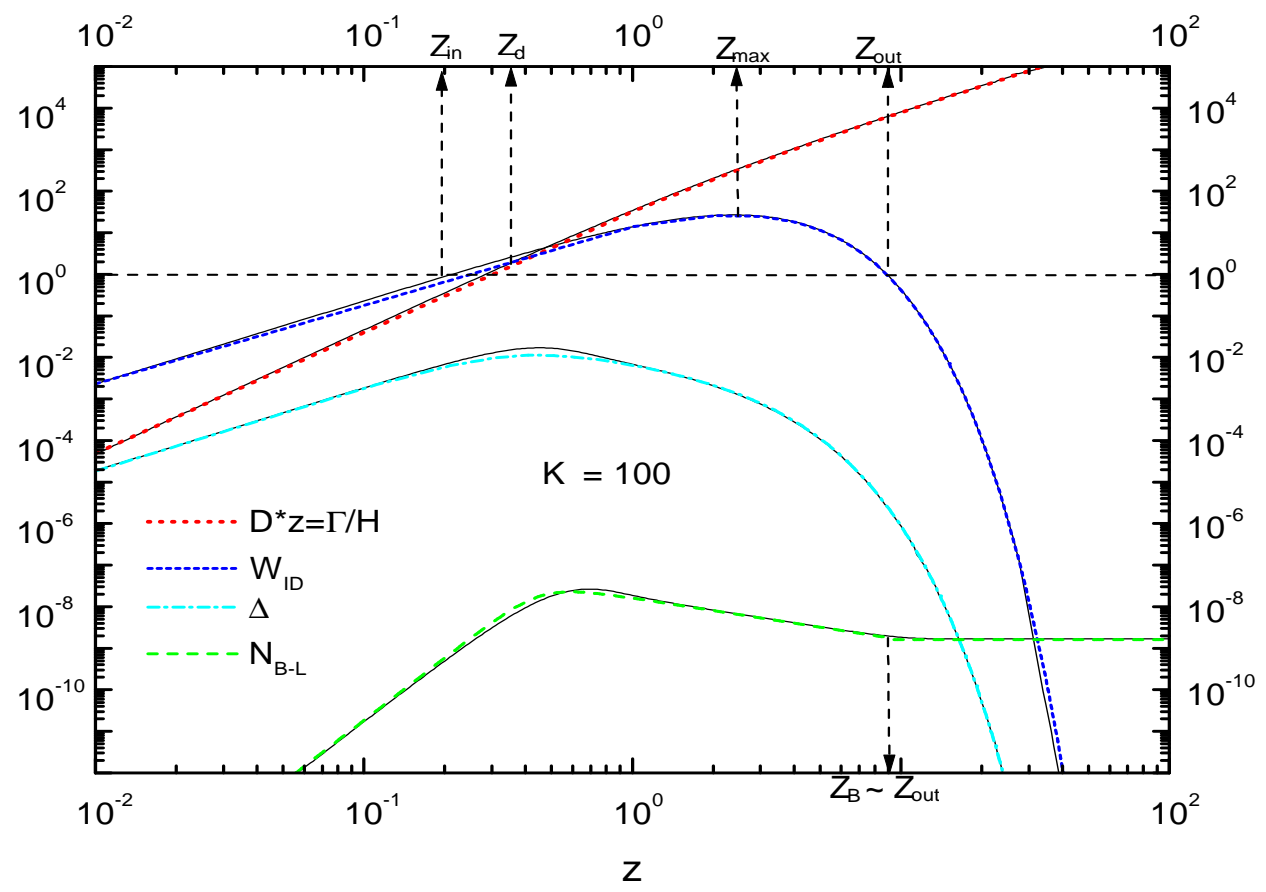

Figure 4: Strong washout: comparison between analytical and numerical (full lines) results. Inverse decays are in equilibrium in the temperature range $z_{\text {in }} \leq z \leq z_{\text {out }} \sim z_{B} ;\left|\varepsilon_{1}\right|=10^{-6}$.

inverse decay and washout terms. Instead of the neutrino abundance the deviation $\Delta(z)$ is shown. The dotted, short dashed, dot-dashed and dashed lines are the approximations Eq. (26) for the $D$ term, Eq. (27) for the $W_{I D}$ term, Eq. (56) for $\Delta(z)$ and Eq. (57) for $\kappa(z)$, respectively. The thin solid lines are the numerical results which agree well with the analytical approximations. The behavior $\kappa(z) \propto 1 / z$ for $z>z_{\mathrm{d}}$ and the freeze-out of $N_{B-L}$ at $z_{\text {out }}$ are clearly visible.

Let us now focus on the final value of the efficiency factor $\kappa_{\mathrm{f}}=\kappa(\infty)$. Note, that for $K \gg 1$ also $z_{\mathrm{B}} \gg 1$, and the condition (53) becomes approximately $W_{I D}\left(z_{\mathrm{B}}\right) \simeq 1$. This means $z_{\mathrm{B}} \simeq z_{\text {out }}$. Hence, the asymmetry produced for $z \leq z_{\text {out }}$ is essentially washed out, while for for $z>z_{\text {out }}$ washout is negligible $\left(W_{I D}<1\right)$. This simple picture will have some interesting consequences and applications.

The integral in Eq. (55) is easily evaluated,

$$
\int_{0}^{\infty} d z^{\prime} \bar{W}_{I D}(z)=\frac{1}{2} z_{\mathrm{B}}(K) K .
$$




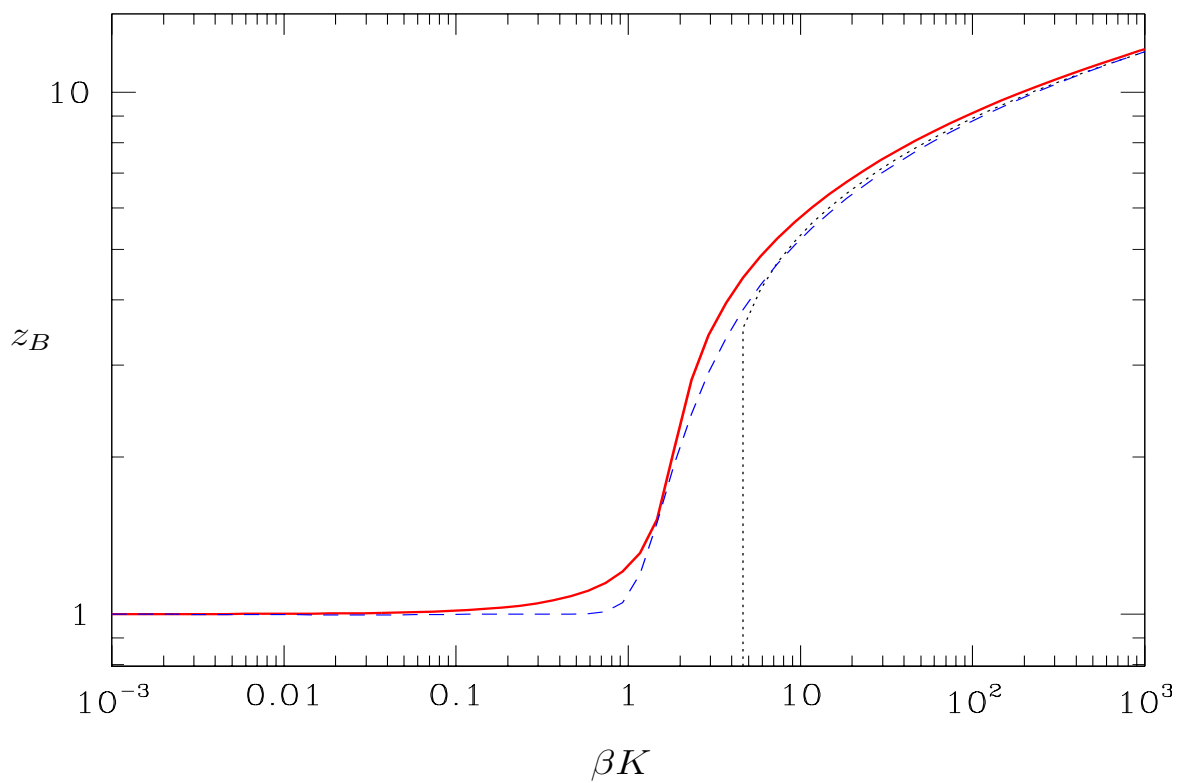

Figure 5: $z_{B}$ as function of the decay parameter $\beta K$. The case studied in this section corresponds to $\beta=1$. The solid red line is the numerical solution of eq. (59), the dotted black line shows the asymptotic solution (60), and the blue dashed line is the interpolation (62).

Using the approximations (18) and (27), the condition (53) for $z_{\mathrm{B}}(K)$ becomes explicitly

$$
\frac{K}{4} z_{\mathrm{B}}(K)^{3} e^{-z_{\mathrm{B}}(K)} \sqrt{1+\frac{\pi}{2} z_{\mathrm{B}}(K)} \simeq z_{\mathrm{B}}(K)-1 .
$$

$z_{B}(K)$ approaches one as $K$ goes to zero ${ }^{2}$. For $K \gg 1$ the solution of Eq. (59) is given by

$$
z_{B}(K) \simeq-\frac{5}{2} W_{-1}\left(-\frac{4}{5 \pi^{1 / 5}} K^{-2 / 5}\right)
$$

where $W_{-1}$ is one of the real branches of the Lambert $W$ function [16]. This result can be approximated by using the asymptotic expansion of $W_{-1}[16,17]$,

$$
z_{B}(K) \simeq \frac{1}{2} \ln \left(\frac{\pi K^{2}}{1024}\left[\ln \left(\frac{3125 \pi K^{2}}{1024}\right)\right]^{5}\right)+\mathcal{O}\left(\frac{\ln (K)}{\ln (\ln (K))}\right)
$$

A rather accurate expression for $z_{B}(K)$ for all values of $K$ is given by the interpolation (cf. Fig. 5),

$$
z_{B}(K) \simeq 1+\frac{1}{2} \ln \left(1+\frac{\pi K^{2}}{1024}\left[\ln \left(\frac{3125 \pi K^{2}}{1024}\right)\right]^{5}\right) .
$$

\footnotetext{
${ }^{2}$ Note that the solution $z_{B}(K)$ of Eq. (53) approaches asymptotically 1.33 for $K \rightarrow 0$. However, in the strong washout regime and also for the extrapolation $K \rightarrow 0$ this difference is irrelevant for $\kappa_{\mathrm{f}}$.
} 


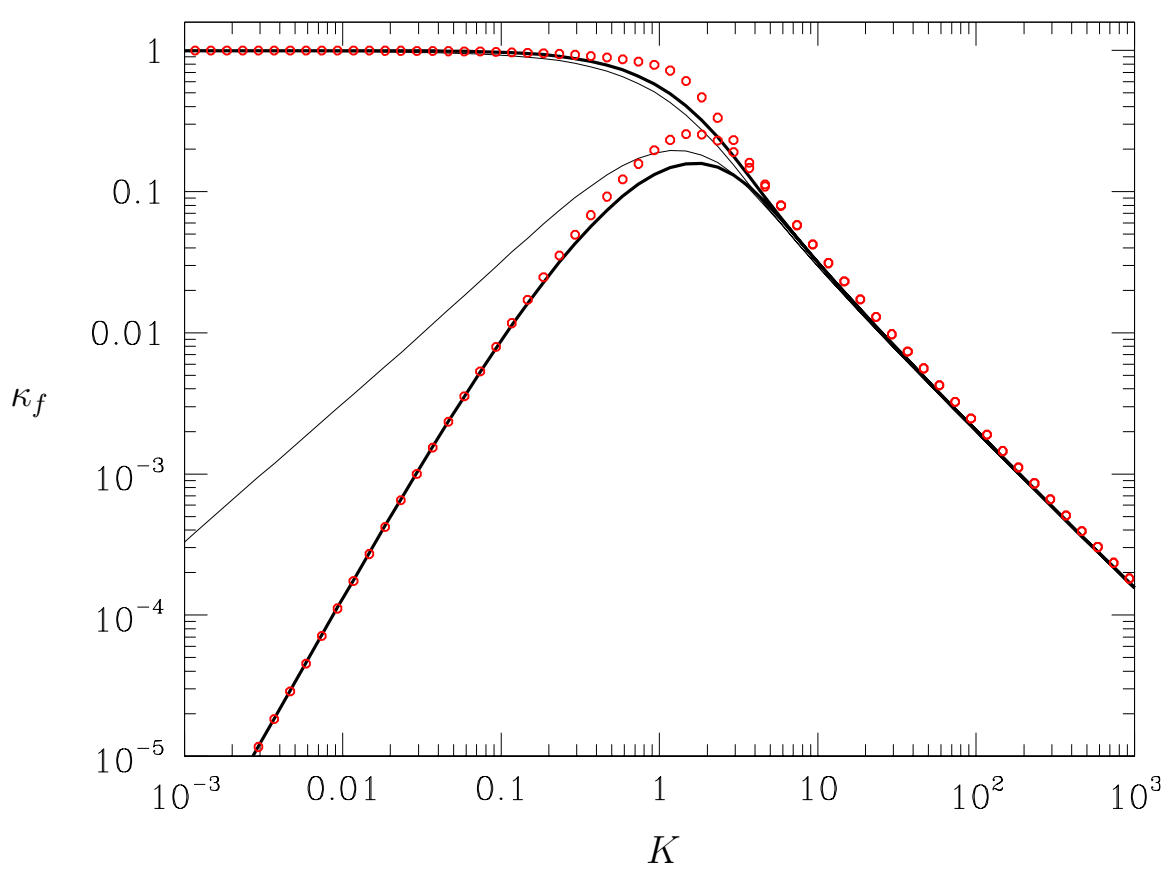

Figure 6: The final efficiency factor $\kappa_{\mathrm{f}}$ as function of the decay parameter $K$ for thermal and dynamical initial $N_{1}$ abundance, respectively. The thick solid lines are the numerical solutions. The thin lines show, for comparison, the numerical solutions of the complete Boltzmann equations including $N_{1}$-top scatterings with an effective Higgs mass $M_{h} / M_{1}=$ 0.1. The red circles represent Eq. (63) for the efficiency factor, evaluated using Eq. (62) for $z_{B}(K)$.

Note the rapid transition from strong to weak washout at $K \simeq 3$.

The final value of the efficiency factor takes the simple form

$$
\kappa_{\mathrm{f}}(K) \simeq \frac{2}{z_{\mathrm{B}}(K) K}\left(1-e^{-\frac{1}{2} z_{\mathrm{B}}(K) K}\right)
$$

This analytical expression for the final efficiency factor, combined with Eq. (62) for $z_{B}(K)$, provides an accurate description of $\kappa_{\mathrm{f}}(K)$, as shown in Fig. 6. Eq. (63) can also be extrapolated into the regime of weak washout, $K \ll 1$, where one obtains $\kappa_{\mathrm{f}}=1$ corresponding to thermal initial abundance, $N_{N_{1}}^{\mathrm{i}}=N_{N_{1}}^{\mathrm{eq}}=3 / 4$. It turns out that also in the transition region Eqs. (63) and (62) provide a rather accurate description, as is evident from Fig. 6. The largest discrepancy between analytical and numerical results is about $30 \%$ around $K \sim 1$. For comparison also the numerical result including scatterings is shown. The difference with respect to the basic 'decay-plus-inverse-decay' picture becomes significant only for $K<1$. 
The above analysis is easily extended to the case where the strength of the washout term $W_{I D}$ is modified to $\beta W_{I D}$. For instance, in the model considered in [3], $B-L$ number changes by two in heavy particle decays, corresponding to $\beta=2$. On the other hand, the heavy particle abundance is not affected by this change. The final efficiency factor is therefore given by

$$
\kappa_{\mathrm{f}}(K) \simeq \frac{2}{z_{B}(K) \beta K}\left(1-e^{-\frac{1}{2} z_{B}(K) \beta K}\right)
$$

where $z_{B}(K)$ is again given by Eq. (59). In the regime $K \gg 1$ our expression for the final efficiency factor can be approximated by

$$
\kappa_{\mathrm{f}}(K) \simeq \frac{2}{z_{B}(K) \beta K} \simeq \frac{1}{1.2 \beta K(\ln \beta K)^{0.8}},
$$

which is very similar to the result ${ }^{3}$ obtained by Kolb and Turner [3].

Comparing the efficiency factor (63) with the solution of the Boltzmann equations including scatterings, as shown in Fig. 6, one arrives at the conclusion that the simple decay-plus-inverse-decay picture represents a very good approximation for leptogenesis in the strong washout regime. As we will see, the difference is essentially negligible within the current theoretical uncertainties.

\subsubsection{Global parametrization}

Given the results of the previous sections it is straightforward to obtain an expression for the efficiency factor for all values of $K$ also in the case of dynamical initial abundance. We first introduce a number density $\bar{N}(K)$ which interpolates between the maximal number densities $N_{\text {eq }}=3 / 4$ and $N(K)=9 \pi K / 16$ (cf. (38)) for strong and weak washout, respectively,

$$
\bar{N}(K)=\frac{N(K)}{\left(1+\sqrt{\frac{N(K)}{N_{\mathrm{eq}}}}\right)^{2}} .
$$

The efficiency factor is in general the sum of a positive and a negative contribution,

$$
\kappa_{\mathrm{f}}(K)=\kappa_{\mathrm{f}}^{+}(K)+\kappa_{\mathrm{f}}^{-}(K) .
$$

Here $\kappa_{\mathrm{f}}^{-}(\mathrm{K})$ is given by $(42)$ for $K \ll 1$. A generalization, accounting for washout also for $z \geq z_{\text {eq }}$, reads

$$
\kappa^{-}(z)=\kappa^{-}\left(z_{\mathrm{eq}}\right) e^{\frac{2}{3} N(K)-\int_{0}^{z} d z^{\prime} W_{\mathrm{ID}}\left(z^{\prime}\right)}
$$

\footnotetext{
${ }^{3}$ Quantitatively, a discrepancy by a factor $\sim 7$ was noted in [18], which is mostly related to the definition of the decay parameter $K$ (cf. Eq. (6)).
} 
This expression extends the validity of the analytical solution to values $K>10^{-2}$ in the case of a dynamical initial abundance. $\kappa_{\mathrm{f}}$ is exponentially suppressed for $K \gg 1$. An interpolation, satisfying the asymptotic behaviors at small and large $K$, is given by

$$
\kappa_{\mathrm{f}}^{-}(K)=-2 e^{-\frac{2}{3} N(K)}\left(e^{\frac{2}{3} \bar{N}(K)}-1\right) .
$$

On the other hand, the expression (63) for $\kappa_{\mathrm{f}}^{+}(K)$, which is valid for $K \gg 1$, has to approach $4 / 3 N(K)$ for $K \ll 1$. These requirements are fulfilled by

$$
\kappa_{\mathrm{f}}^{+}(K)=\frac{2}{z_{B}(K) K}\left(1-e^{-\frac{2}{3} z_{B}(K) K \bar{N}(K)}\right) .
$$

Equations (66), (68) and (69), together with the interpolation (62) for $z_{B}(K)$, yield an accurate description of the efficiency factor $\kappa_{\mathrm{f}}(K)$ for all values of $K$, as demonstrated by Fig. 6.

This result is a good starting point for obtaining an analytic description of the efficiency factor for the full problem. In the following sections we shall go beyond the simple decay-and-inverse-decay picture and include other processes step by step.

\section{The scattering term}

\subsection{Analytic approximations}

The scattering term $S$ and the related washout contribution $W_{\Delta L=1}$ arise from two different classes of Higgs and lepton mediated inelastic scatterings involving the top quark $(t)$ and gauge bosons $(A)$,

$$
S=S^{t}+S^{A}
$$

Their main effect is to enhance the neutrino production and thus the efficiency factor for $\widetilde{m}_{1}<m_{*}$. Further, they also contribute to the washout term, which leads to a correction of the efficiency factor for $\widetilde{m}_{1}>m_{*}$, i.e. in the strong washout regime. Since the scattering processes are specific to leptogenesis we shall use in this section mostly the variable $\widetilde{m}_{1} \geq m_{1}$ [19] rather than $K$. Top quark and gauge boson scattering terms are expected to be of similar size. However, the reaction densities for the gauge boson processes are presently controversial $[11,12]$. We shall therefore discuss these processes in detail elsewhere. We shall also neglect the scale dependence of the top-Yukawa coupling, which reduces the size of $S^{t}$, since this decrease of $S$ will be partially compensated by $S^{A}$.

The term $S^{t}$ is again the sum of two terms arising from the s-channel processes $N_{1} l \leftrightarrow$ $t q$ and the t-channel processes $N_{1} t \leftrightarrow l q, N_{1} \bar{q} \leftrightarrow l \bar{t}$,

$$
S^{t}=2 S_{\phi, s}+4 S_{\phi, t} .
$$


The scattering terms are defined as usual in terms of scattering rates and expansion rate,

$$
S_{\phi, s(t)}=\frac{\Gamma_{\phi, s(t)}^{\left(N_{1}\right)}}{H z}
$$

and introducing the functions $f_{\phi, s(t)}(z)$ (cf. appendix B) it is possible to write

$$
S^{t}=\frac{K_{S}}{6}\left(f_{\phi, s}(z)+2 f_{\phi, t}(z)\right)
$$

here we have introduced the ratio

$$
K_{S}=\frac{\widetilde{m}_{1}}{m_{*}^{s}}
$$

with

$$
m_{*}^{s}=\frac{4 \pi^{2}}{9} \frac{g_{N_{1}} v^{2}}{m_{t}^{2}} m_{*} \simeq 10 m_{*} .
$$

At high temperatures, $z \ll 1$, the functions $f_{\phi, s(t)}$ have the following asymptotic form,

$$
\begin{aligned}
f_{\phi, s}(z) & \simeq 2\left[1-z^{2}\left(\ln \left(\frac{2}{z}\right)-\gamma_{E}\right)\right] \\
f_{\phi, t}(z) & \simeq 2\left[1+\frac{z^{2}}{2} \ln \left(\frac{M_{1}}{M_{h}}\right)\left(\ln \left(\frac{2}{z}\right)-\gamma_{E}\right)\right]
\end{aligned}
$$

In Fig. 7 we have plotted the rates $S+D$ and $D$ as function of $z$ for $K_{S}=1$, i.e. $\widetilde{m}_{1}=m_{*}^{s}$. For values $z<2$ the sum $S+D$ is dominated by the scattering rate $S$, while for $z>2$ the decay rate $D \simeq K z$ dominates. A simple analytic approximation for the sum $D+S$ is given by

$$
D+S \simeq K_{S}\left[1+\ln \left(\frac{M_{1}}{M_{h}}\right) z^{2} \ln \left(1+\frac{a}{z}\right)\right],
$$

where

$$
a=\frac{K}{K_{S} \ln \left(M_{1} / M_{h}\right)}=\frac{8 \pi^{2}}{9 \ln \left(M_{1} / M_{h}\right)} .
$$

Here we have introduced the Higgs mass $M_{h}$ to cut off the infrared divergence of the t-channel process. As Fig. 7 illustrates, the approximation (78) agrees well with the numerical result for $M_{h} / M_{1}=10^{-5}$ as well as $M_{h} / M_{1}=10^{-1}$. Note that the latter value corresponds to the thermal Higgs mass, $M_{h} \simeq 0.4 T$, at the baryogenesis temperature $T_{B}$ in the strong washout regime.

The washout term induced by the $\Delta L=1$ scattering processes is again the sum of sand t-channel contributions,

$$
W_{\Delta L=1}=W_{\phi, s}+2 W_{\phi, t}
$$




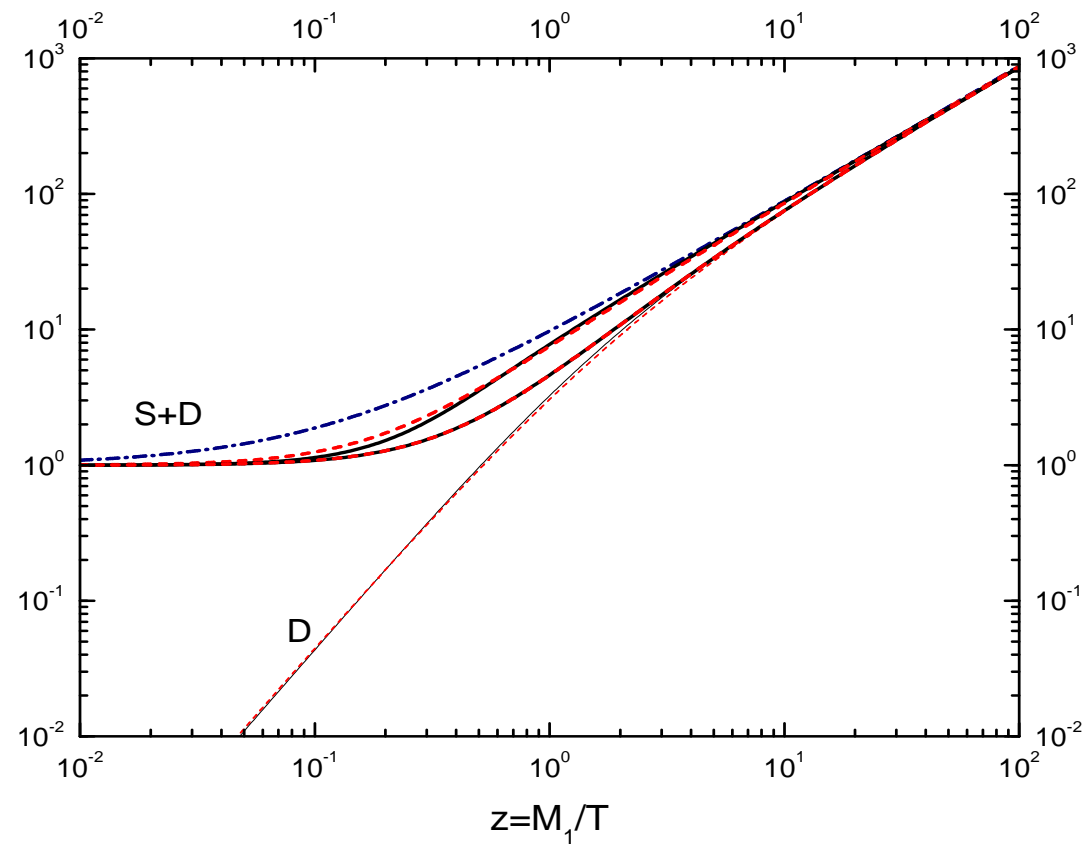

Figure 7: The rates $S+D$ and $D$ are shown as function of $z$ for $K_{S}=1$, i.e. $\widetilde{m}_{1}=m_{\star}^{s}$. The dash-dotted line is the simple approximation $S+D \simeq K_{S}+K z$, while the two short dashed lines represent Eq. (78) for $M_{h} / M_{1}=10^{-5}$ (higher) and $M_{h} / M_{1}=10^{-1}$ (lower), to be compared with the numerical results (thick solid lines). The dotted line shows the expression (18) for $D$, to be compared with the numerical result for $D$ (solid line).

The washout rates are directly related to the scattering rates (72),

$$
\begin{aligned}
W_{\phi, t} & =\frac{\Gamma_{\phi, t}^{l}}{H z}=\frac{N_{N_{1}}^{\mathrm{eq}}}{N_{l}^{\mathrm{eq}}} S_{\phi, t} \\
W_{\phi, s} & =\frac{N_{N_{1}}}{N_{N_{1}}^{\mathrm{eq}}} \frac{\Gamma_{\phi, s}^{l}}{H z}=\frac{N_{N_{1}}^{\mathrm{eq}}}{N_{l}^{\mathrm{eq}}} \frac{N_{N_{1}}}{N_{N_{1}}^{\mathrm{eq}}} S_{\phi, s} .
\end{aligned}
$$

Using Eq. (22) for the equilibrium number densities one obtains

$$
W_{\Delta L=1}=2 W_{I D} \frac{1}{D}\left(\frac{N_{N_{1}}}{N_{N_{1}}^{\mathrm{eq}}} S_{\phi, s}+2 S_{\phi, t}\right) .
$$

The washout rate including inverse decays is then given by

$$
\begin{aligned}
W_{0} & =W_{I D}+W_{\Delta L=1} \\
& =W_{I D}\left(1+\frac{1}{D}\left(2 \frac{N_{N_{1}}}{N_{N_{1}}^{\mathrm{eq}}} S_{\phi, s}+4 S_{\phi, t}\right)\right) .
\end{aligned}
$$


This is the total washout rate as long as $\Delta W$, the off-shell contribution from heavy neutrinos, can be neglected. This is justified for sufficiently small values of $M_{1}$ (cf. sect. 4).

\subsection{Dynamical initial abundance}

We can now calculate the production of heavy neutrinos and study how the efficiency factor gets enhanced by the presence of the scattering term. We again define a value $z_{\text {eq }}$ by the condition (31),

$$
N_{N_{1}}\left(z_{\text {eq }}\right)=N_{N_{1}}^{\mathrm{eq}}\left(z_{\text {eq }}\right) .
$$

For $z<z_{\text {eq }}$ the number density can be obtained by integrating the equation

$$
\frac{d N_{N_{1}}}{d z} \simeq(D+S) N_{N_{1}}^{\mathrm{eq}}>0 .
$$

The result is given by the expression $\left(z \leq z_{\mathrm{eq}}\right)$

$$
N_{N_{1}}(z)=\frac{3}{8} K_{S}\left[\mathcal{I}_{A}(z)+\ln \left(\frac{M_{1}}{M_{h}}\right) \mathcal{I}_{B}(z)\right] .
$$

Here the first integral is given by

$$
\mathcal{I}_{A}(z)=\int_{0}^{z} d z^{\prime} z^{\prime 2} K_{2}\left(z^{\prime}\right) \simeq \frac{3 \pi z^{3}}{\left[(9 \pi)^{c}+\left(2 z^{3}\right)^{c}\right]^{1 / c}}+z^{3} K_{2}(z),
$$

where we have used the approximation Eq. (39). The second integral can be expressed as

$$
\begin{aligned}
\mathcal{I}_{B}(z) & =\int_{0}^{z} d z^{\prime} z^{\prime 4} \ln \left(1+\frac{a}{z^{\prime}}\right) K_{2}\left(z^{\prime}\right) \\
& \simeq 2 \int_{0}^{1} d z^{\prime} z^{\prime 2} \ln \left(1+\frac{a}{z^{\prime}}\right)+a \int_{1}^{z} d z^{\prime} z^{\prime 3} K_{2}\left(z^{\prime}\right) \\
& \simeq \frac{2}{3}\left(\left(1+a^{3}\right) \ln (1+a)-a^{3} \ln a-a^{2}+\frac{1}{2} a\right)+a K_{3}(1)-a z^{3} K_{3}(z) .
\end{aligned}
$$

The value $z_{\text {eq }}$ can now be determined by setting $N_{N_{1}}$, as determined from Eqs. (86), (87) and (88), equal to $N_{N_{1}}^{\text {eq }}$. Using an approximate form for $K_{3}$, one obtains an equation similar to Eq. (59), as described in appendix B. This yields a good approximation for $z_{\mathrm{eq}}$ in the case $\widetilde{m}_{1}<m_{*}$.

\subsubsection{Weak washout regime}

Consider now the case of weak washout, $\widetilde{m}_{1} \ll m_{*} \simeq 10^{-3} \mathrm{eV}$, which implies $z_{\text {eq }} \gg 1$. For $z>z_{\text {eq }}$, decays dominate over inverse decays,

$$
\frac{d N_{N_{1}}}{d z} \simeq-(D+S) N_{N_{1}}<0 .
$$


Using $D+S \simeq K z$, valid for $z \gg a$ (cf. (78) and fig. 7), this yields for the number density the simple expression

$$
\begin{aligned}
N_{N_{1}}(z) & \simeq N_{N_{1}}^{\mathrm{eq}}\left(z_{\mathrm{eq}}\right) e^{-\int_{\mathrm{eq}_{\mathrm{eq}}}^{z} d z^{\prime}(S+D)} \\
& \simeq N_{N_{1}}^{\mathrm{eq}}\left(z_{\mathrm{eq}}\right) e^{-\frac{K}{2}\left(z^{2}-z_{\mathrm{eq}}^{2}\right)}
\end{aligned}
$$

In Fig. 8 the solution $N_{N_{1}}(z)$ is shown for $\widetilde{m}_{1}=10^{-5} \mathrm{eV}$. The analytical solution agrees well with the numerical result. We also make a comparison with the result already displayed in Fig. 3, where the $\mathrm{S}$ term is neglected. As expected, the presence of the $\mathrm{S}$ term enhances the density $N_{N_{1}}$ at $z=z_{\text {eq }}$. Moreover the comparison illustrates the strong sensitivity of the efficiency factor in the case $\widetilde{m}_{1} \ll m_{*}$, not only to the initial conditions, but also to the theoretical description. A difference in $N_{1}$ abundance by less than a factor of two at $z_{\text {eq }}$ corresponds to final efficiency factors which differ by two orders of magnitude. This is due to delicate cancellations between the positive and the negative contribution to the efficiency factor and is a source of large theoretical uncertainties in the small $\widetilde{m}_{1}$ regime.

We can now calculate the efficiency factor. In sect. 2.3.1 we have seen that in the absence of scatterings the inclusion of the small washout term was necessary to create an asymmetry between the negative contribution, $\kappa^{-}$, and the positive one, $\kappa^{+}$, in order to have a non-zero final value $\kappa_{f}$. Given the $S$ term one can neglect washout to first approximation. From Eq. (8) one then obtains

$$
\kappa(z)=-\frac{4}{3} \int_{z_{\mathrm{i}}}^{z} d z^{\prime} j^{-1} \frac{d N_{N_{1}}}{d z^{\prime}}
$$

where (cf. (18), (78))

$$
j(z)=\frac{D+S}{D} \simeq\left[\frac{z}{a} \ln \left(1+\frac{a}{z}\right)+\frac{K_{S}}{K z}\right]\left(1+\frac{15}{8 z}\right) .
$$

Due to the $\mathrm{S}$ term we now have $\kappa_{\mathrm{f}} \neq \frac{4}{3} N_{N_{1}}^{\mathrm{i}}$, although washout is neglected. The reason is quite clear: as in the case without scatterings, the asymmetry is changed only by decays and inverse decays; however, the number of decaying neutrinos at $z_{\text {eq }}$ is now larger because of the additional production due to scatterings. To first approximation we can thus calculate the efficiency factor neglecting washout.

For $z \leq z_{\text {eq }}$ one obtains (cf. (39)),

$$
\kappa^{-}(z)=-\frac{4}{3} \int_{z_{\mathrm{i}}}^{z} d z^{\prime} D N_{N_{1}}^{\mathrm{eq}} \simeq-\frac{3 \pi K z^{3}}{\left[(18 \pi)^{c}+\left(4 z^{3}\right)^{c}\right]^{1 / c}} .
$$




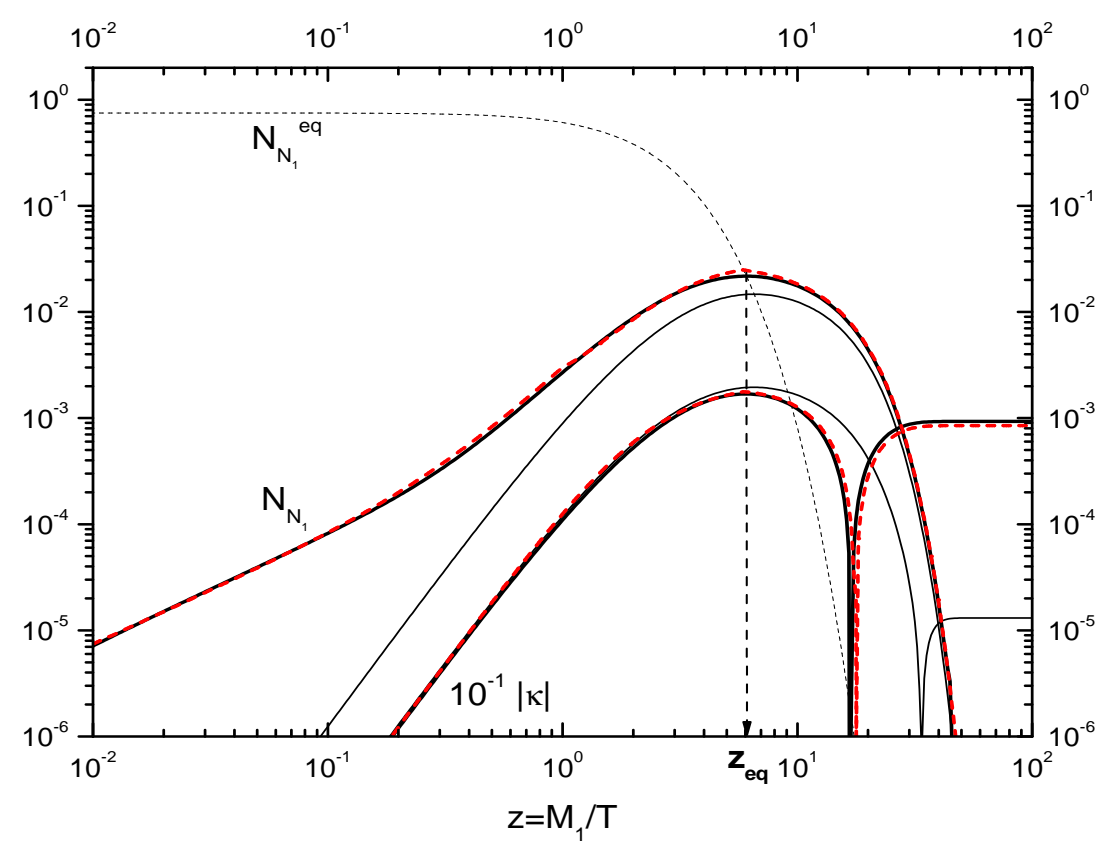

Figure 8: Effect of scatterings on neutrino production for $\widetilde{m}_{1}=10^{-5} \mathrm{eV}$. The numerical results have been calculated for $\Delta W=0$ and $M_{h} / M_{1}=10^{-5}$ (thick solid lines). The short-dashed lines are the analytical solutions for $N_{N_{1}}$ (Eqs. (86) and (90)) and for $\kappa(z)$ (Eqs. (93) and (94)), with $M_{h} / M_{1}=10^{-5}$. For comparison we also show the result where scatterings are neglected (thin solid lines).

For $z>z_{\text {eq }}$ one has $j \simeq$ const. (cf. Fig. 7); Eq. (91) the yields the simple result,

$$
\kappa(z)=\frac{4}{3}\left(N_{N_{1}}\left(z_{\mathrm{eq}}\right) j^{-1}\left(z_{\mathrm{eq}}\right)-N_{N_{1}}(z) j^{-1}(z)\right)+\kappa^{-}\left(z_{\mathrm{eq}}\right),
$$

which is shown in Fig. 8 for $\widetilde{m}_{1}=10^{-5}$ and $M_{h} / M_{1}=10^{-5}$ (short dashed line); it agrees reasonably well with the corresponding numerical solution (solid line). The analytical solution somewhat overestimates $\left|\kappa_{\mathrm{f}}^{-}\right|$; correspondingly, the final value $\kappa_{f}$ is underestimated.

The analytical solution explains why the final value of the efficiency factor, $\kappa_{\mathrm{f}}$, is proportional to $\widetilde{m}_{1}$,

$$
\kappa_{\mathrm{f}} \simeq \frac{4}{3}\left[N_{N_{1}}\left(z_{\mathrm{eq}}\right) j^{-1}\left(z_{\mathrm{eq}}\right)-\tilde{N}(K)\right] \propto \widetilde{m}_{1}
$$

where

$$
\widetilde{N}(K)=\frac{2 N(K) z_{\mathrm{eq}}^{3}}{\left((9 \pi)^{c}+\left(2 z_{\mathrm{eq}}^{3}\right)^{c}\right)^{1 / c}},
$$




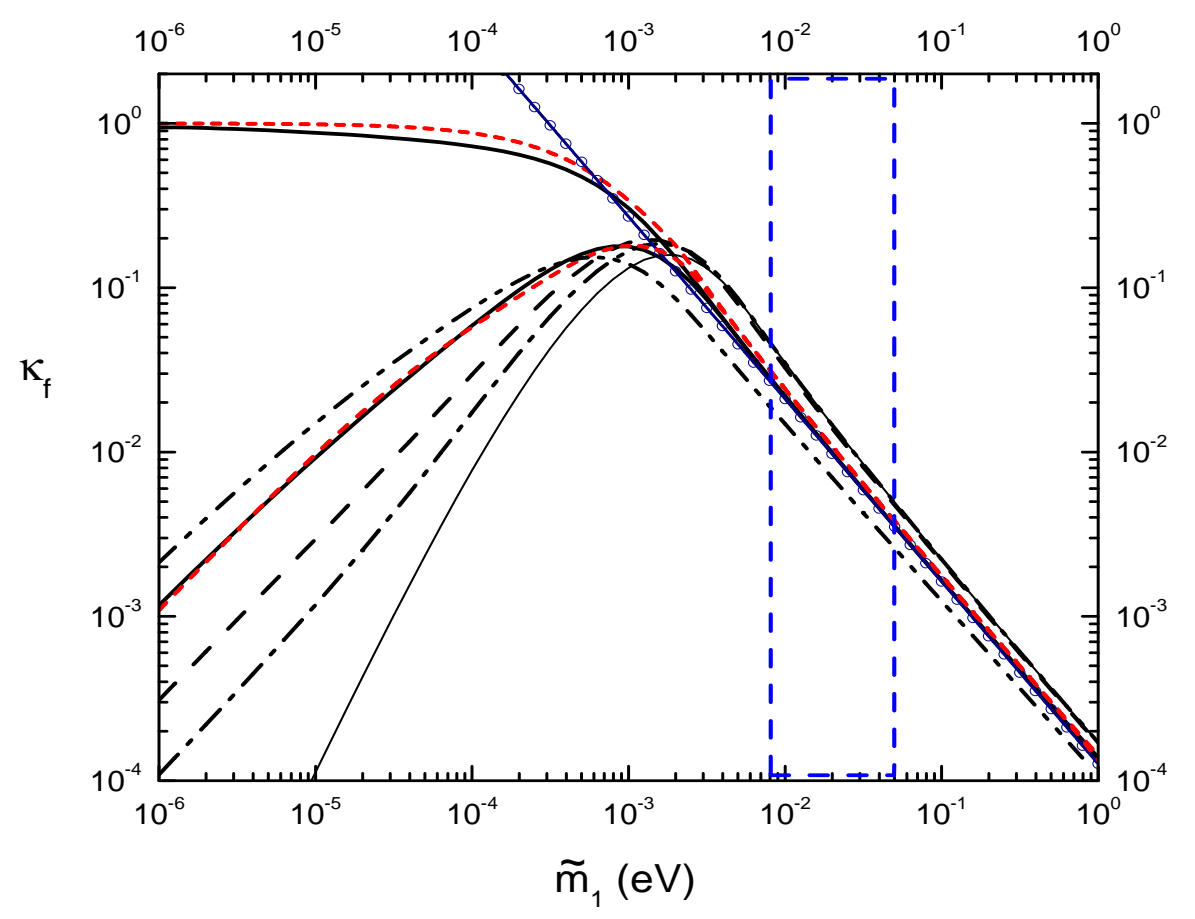

Figure 9: The final efficiency factor when scatterings are included. The numerical results are shown for $M_{h} / M_{1}=10^{-10}, 10^{-5}, 10^{-1}, 1$ (dot-dot-dash, solid, dashed and dot-dashed line respectively). The thin dashed line is the simple result from the decay-plus-inverse-decay picture when scatterings are neglected. The short-dashed lines are the analytical results in the case of thermal initial abundance (Eq. (107) with $j\left(z_{B}\right) \rightarrow j^{2}\left(z_{B}\right)$ in the exponential) and zero initial abundance (Eqs. $\left.(110)+(109), M_{h} / M_{1}=10^{-5}\right)$. The circled line is the power law fit (108). The dashed box indicates the range $\left(m_{\mathrm{sol}}, m_{\mathrm{atm}}\right)$.

with $N(K)=9 \pi K / 16$. Contrary to the case discussed in sect. 2.3.1, for which $N_{N_{1}}\left(z_{\text {eq }}\right) \simeq$ $\widetilde{N}(K) \simeq \bar{N}(K)$ and $j=1, N_{N_{1}}\left(z_{\text {eq }}\right)$ and $\widetilde{N}(K)$ are now different. Hence, there is no cancellation of terms $\mathcal{O}\left(\widetilde{m}_{1}\right)$ between $\kappa^{+}$and $\kappa^{-}$.

The expression (95) for the final efficiency factor fails for effective neutrino masses $\widetilde{m}_{1}>10^{-5} \mathrm{eV}$. Including washout mainly reduces the negative contribution $\kappa^{-}$and thereby enhances the final value of the efficiency factor. Eq. (93) is then changed into

$$
\kappa_{\mathrm{f}}^{-}=-\frac{4}{3} \int_{z_{\mathrm{i}}}^{\infty} d z^{\prime} D N_{N_{1}}^{\mathrm{eq}} e^{-\int_{z^{\prime}}^{\infty} d z^{\prime \prime} W_{0}\left(z^{\prime \prime}\right)} .
$$

For $z<z_{\text {eq }}$ one has $N_{N_{1}}<N_{N_{1}}^{\mathrm{eq}}$, and the washout rate becomes (cf. (84))

$$
W_{0}(z) \simeq W_{I D}(z) \frac{D+4 S_{\phi, t}}{D}
$$


From the expression (78) for $D+S$ one obtains

$$
\begin{aligned}
D+4 S_{\phi, t} & \simeq K_{S}\left(\frac{2}{3}+\ln \left(\frac{M_{1}}{M_{h}}\right) z^{2} \ln \left(1+\frac{a}{z}\right)\right) \\
& \simeq \frac{2}{3} K_{s}+K z
\end{aligned}
$$

here the last approximation requires $a \ll 1$. Together with Eq. (18) this yields

$$
W_{0}(z) \simeq W_{I D}(z)\left(1+\frac{\alpha}{z}\right)
$$

where the coefficient $\alpha$ is given by

$$
\alpha=\frac{2 K_{S}}{3 K}+\frac{15}{8}
$$

Since $W_{I D} / z$ is a total derivative, one obtains for the efficiency factor,

$$
\begin{aligned}
\kappa_{\mathrm{f}}^{-} & =-2 \int_{z_{\mathrm{i}}}^{\infty} d z^{\prime} W_{I D}\left(z^{\prime}\right) e^{-\frac{1}{4} K \alpha z^{2} K_{2}\left(z^{\prime}\right)} e^{-\int_{z^{\prime}}^{\infty} d z^{\prime \prime} W_{I D}\left(z^{\prime \prime}\right)} \\
& \simeq-2 e^{-\frac{1}{2} K \alpha} \int_{z_{\mathrm{i}}}^{\infty} d z^{\prime} W_{I D}\left(z^{\prime}\right) e^{-\int_{z^{\prime}}^{\infty} d z^{\prime \prime} W_{I D}\left(z^{\prime \prime}\right)}
\end{aligned}
$$

where we have used $z^{2} K_{2}(z) \simeq 2$ for $z \lesssim 1$. Except for the exponential pre-factor, this yields the result obtained in sect. 2.3.1 for decays and inverse decays (cf. (42)) when the wash-out at $z>z_{\text {eq }}$ is neglected

$$
\kappa_{\mathrm{f}}^{-}=-2 e^{-\frac{1}{2} K \alpha}\left(1-e^{-\frac{2}{3} N(K)}\right) .
$$

The case without scatterings is recovered for $\alpha=0$.

\subsubsection{Strong washout regime}

In the case of strong washout, $K \gg 1$, the density of heavy neutrinos follows closely the equilibrium abundance, and one can obtain an analytical solution repeating the discussion in sect. 2.3.2. The efficiency factor is now given by

$$
\kappa(z)=-\frac{4}{3} \int_{z_{\mathrm{in}}}^{z} d z^{\prime} j^{-1} \frac{d N_{N_{1}}^{\mathrm{eq}}}{d z^{\prime}} e^{-\int_{z^{\prime}}^{z} d z^{\prime \prime} W_{0}\left(z^{\prime \prime}\right)} .
$$

Using $N_{N_{1}} / N_{N_{1}}^{\mathrm{eq}} \simeq 1$ in the washout rate one obtains (cf. (84)),

$$
W_{0} \simeq W_{I D} j
$$


In this way one finds for the efficiency factor

$$
\begin{aligned}
\kappa(z) & =\frac{2}{K} \int_{z_{\mathrm{i}}}^{z} d z^{\prime} \frac{1}{z^{\prime} j\left(z^{\prime}\right)} W_{I D}\left(z^{\prime}\right) e^{-\int_{z^{\prime}}^{z} d z^{\prime \prime} W_{I D}\left(z^{\prime \prime}\right) j\left(z^{\prime \prime}\right)} \\
& \equiv \int_{z_{\mathrm{i}}}^{z} d z^{\prime} e^{-\psi_{S}\left(z^{\prime}, z\right)} .
\end{aligned}
$$

As in sect. 2.3.2, the dominant contribution to the integral arises from a region around a value $z_{B} \gg 1$ where $\psi_{S}\left(z^{\prime}, z\right)$ has a minimum. Since $D+S \simeq K_{S}+K z$ for large $z$, the value $z_{B}$ is again given by Eq. (53) up to corrections $\mathcal{O}\left(K_{S} /\left(K z_{0}^{2}\right)\right)$. Replacing now $W_{I D}(z)$ by $W_{I D}(z) z_{B} j\left(z_{B}\right) /(z j(z))$ in the exponent of the integrand and by $W_{I D}(z) j(z) / j\left(z_{B}\right)$ in the pre-factor, respectively, one obtains for the final efficiency factor the approximate solution,

$$
\kappa_{\mathrm{f}}=\frac{2}{z_{B} K j\left(z_{B}\right)^{2}}\left(1-e^{-\frac{1}{2} z_{B} K j\left(z_{B}\right)}\right) .
$$

This extends Eq. (64) to the case where scatterings are included.

Note, that at small $K$ the efficiency factor (107) does not approach one, the value corresponding to thermal initial abundance. However, any initial abundance can be reproduced by adjusting the exponent in Eq. (107). Replacing $j\left(z_{B}\right)$ by $j\left(z_{B}\right)^{2}$ leaves Eq. (107) essentially unchanged at large $K$, whereas at small $K$ one has $\kappa_{\mathrm{f}} \simeq 1$ corresponding to thermal initial abundance. The result is shown in Fig. 9 (short-dashed line) and compared with numerical results for different values of $M_{\mathrm{h}} / M_{1}$. In the strong washout regime, $\widetilde{m}_{1} \gg m_{*}$, and for $M_{h} / M_{1}=10^{-5}$, the analytical and numerical results agree within $10 \%$. Since the strong washout regime is most interesting with respect to neutrino mass models, this is one of the most relevant results of this paper.

For practical purposes it is interesting to note that, within the current theoretical uncertainties, the efficiency factor for $\widetilde{m}_{1}>m_{*}$ is given by the simple power law,

$$
\kappa_{\mathrm{f}} \simeq(2 \pm 1) \times 10^{-2}\left(\frac{0.01 \mathrm{eV}}{\widetilde{m}_{1}}\right)^{1.1 \pm 0.1}
$$

The quoted uncertainties represent, approximately, the range visible in Fig. 9 for large $\widetilde{m}_{1}$. It is limited from above by the thin solid line corresponding to decays plus inverse decays and from below by the dot-dot-dashed line where scatterings are included with the extremely small ratio $M_{h} / M_{1}=10^{-10}$. Note that $M_{h} / M_{1}=0.1$ corresponds to a thermal Higgs mass, $M_{h} \simeq 0.4 T$, at the baryogenesis temperature $T_{B} \simeq M_{1} / 5$.

\subsubsection{Global parametrization}

As in sect. 2.3.3 we can now obtain an expression for the efficiency factor for all values of $K$ by interpolating between the two regimes of small $K$ and large $K$. We shall use the 
number density $\tilde{N}(K)$ (cf. (96)) and the interpolation (62) for $z_{B}(K)$, which is related to the baryogenesis temperature by $T_{B}=M_{1} / z_{B}$.

The efficiency factor is the sum of a positive and a negative contribution,

$$
\kappa_{\mathrm{f}}(K)=\kappa_{\mathrm{f}}^{+}(K)+\kappa_{\mathrm{f}}^{-}(K)
$$

Here $\kappa_{\mathrm{f}}^{-}(K)$ differs from Eq. (68) just by the exponential pre-factor induced by the scatterings (cf. (103)), which yields

$$
\kappa_{\mathrm{f}}^{-}(K)=-2 e^{-\frac{2}{3}\left(N(K)+\frac{3}{4} K \alpha\right)}\left(e^{\frac{2}{3} \tilde{N}(K)}-1\right) .
$$

The expression (107) for $\kappa_{\mathrm{f}}^{+}(K)$, which is valid for $K \gg 1$, has to approach Eq. (95) for $K \ll 1$. An interpolating function is given by

$$
\kappa_{\mathrm{f}}^{+}(K)=\frac{2}{z_{B}(K) K j^{2}\left(z_{B}\right)}\left(1-e^{-\frac{2}{3} z_{B}(K) K j^{2}\left(z_{B}\right) N_{N_{1}}\left(z_{\mathrm{eq}}\right) j^{-1}\left(z_{\mathrm{eq}}\right)}\right) .
$$

Eq. (69) is recovered for $j=1$ and $S=0$. The sum $\kappa_{\mathrm{f}}=\kappa_{\mathrm{f}}^{+}+\kappa_{\mathrm{f}}^{-}$is shown in Fig. 9 for $M_{h} / M_{1}=10^{-5}$. The agreement with the numerical result is very good. Including washout yields a description which correctly interpolates between the weak washout regime, $\widetilde{m}_{1} \ll$ $m_{*}$ and the strong washout regime, $\widetilde{m}_{1} \gg m_{*}$.

\subsection{Lower bounds on $M_{1}$}

The results for the efficiency factor are easily translated into theoretical predictions for the observed baryon-to-photon ratio using the relation (10). The theoretical prediction has to be compared with the results from WMAP [20] combined with the Sloan Digital Sky Survey [21], $\Omega_{b} h^{2}=0.023 \pm 0.001$, corresponding to

$$
\eta_{B}^{C M B}=(6.3 \pm 0.3) \times 10^{-10}
$$

The comparison yields the required $C P$ asymmetry in terms of the baryon-to-photon ratio and the efficiency factor $\kappa_{\mathrm{f}}$ (cf. (10)),

$$
\varepsilon_{1}^{C M B}=\frac{\eta_{B}^{C M B}}{d \kappa_{\mathrm{f}}} \simeq 6.3 \times 10^{-8}\left(\frac{\eta_{B}^{C M B}}{6 \times 10^{-10}}\right) \kappa_{\mathrm{f}}^{-1} .
$$

The $C P$ asymmetry $\varepsilon_{1}$ can be written as product of a maximal asymmetry and an effective leptogenesis phase $\delta_{L}[22]$,

$$
\varepsilon_{1}=\varepsilon_{1}^{\max } \sin \delta_{L}
$$


The connection between the leptogenesis phase and other $C P$ violating observables is an important topic of current research [23]. The maximal $C P$ asymmetry $\varepsilon_{1}^{\max }$ depends in general on $M_{1}, \widetilde{m}_{1}$ and, via the light neutrino masses $m_{i}$, on the absolute neutrino mass scale $\bar{m}$ [10]. For given light neutrino masses, i.e. fixed $m_{1}$ and $m_{3}, \varepsilon_{1}$ is maximized in the limit $m_{1} / \widetilde{m}_{1} \rightarrow 0$, for which one obtains [24],

$$
\varepsilon_{1}^{\max }\left(M_{1}, \bar{m}\right)=\frac{3}{16 \pi} \frac{M_{1}}{v^{2}}\left(m_{3}-m_{1}\right) .
$$

This expression reaches its maximum for fully hierarchical neutrinos, corresponding to $m_{1}=0$ and $m_{3}=m_{\text {atm }} \equiv \sqrt{\Delta m_{\text {atm }}^{2}}$.

Neutrino oscillation experiments give for atmospheric neutrinos [25, 26]

$$
\Delta m_{\mathrm{atm}}^{2}=(2.6 \pm 0.4) \times 10^{-3} \mathrm{eV}^{2},
$$

and for solar neutrinos [27]

$$
\Delta m_{\mathrm{sol}}^{2} \simeq\left(7.1_{-0.6}^{+1.2} \times 10^{-5}\right) \mathrm{eV}^{2}
$$

implying

$$
m_{\mathrm{atm}}=(0.051 \pm 0.004) \mathrm{eV} .
$$

Thus, apart the small experimental error, $m_{\mathrm{atm}}$ is a fixed parameter and the resulting maximal asymmetry depends uniquely on $M_{1}$ [24],

$$
\varepsilon_{1}^{\max }\left(M_{1}\right)=\frac{3}{16 \pi} \frac{M_{1} m_{\mathrm{atm}}}{v^{2}} \simeq 10^{-6}\left(\frac{M_{1}}{10^{10} \mathrm{GeV}}\right)\left(\frac{m_{\mathrm{atm}}}{0.05 \mathrm{eV}}\right) .
$$

The maximal $C P$ asymmetry, or equivalently the maximal leptogenesis phase, corresponds to a maximal baryon asymmetry $\eta_{B}^{\max }$. The $\mathrm{CMB}$ constraint $\eta_{B}^{\max } \geq \eta_{B}^{\mathrm{CMB}}$, together with Eq. (118), then yields a lower bound on the heavy neutrino mass $M_{1}$,

$$
\begin{aligned}
M_{1}>M_{1}^{\mathrm{min}} & =\frac{1}{d} \frac{16 \pi}{3} \frac{v^{2}}{m_{\mathrm{atm}}} \frac{\eta_{B}^{C M B}}{\kappa_{\mathrm{f}}} \\
& \simeq 6.4 \times 10^{8} \mathrm{GeV}\left(\frac{\eta_{B}^{C M B}}{6 \times 10^{-10}}\right)\left(\frac{0.05 \mathrm{eV}}{m_{\mathrm{atm}}}\right) \kappa_{\mathrm{f}}^{-1} .
\end{aligned}
$$

Note that the bound depends on the combination $\eta_{B}^{C M B} / m_{\text {atm }}$ whose error, after the WMAP result, receives a similar contribution both from $\eta_{B}^{C M B}$ and $m_{\text {atm }}$ such that

$$
M_{1}^{\mathrm{min}}\left(\widetilde{m}_{1}\right)=(6.6 \pm 0.8) \times 10^{8} \mathrm{GeV} \kappa_{\mathrm{f}}^{-1}\left(\widetilde{m}_{1}\right) \gtrsim 4 \times 10^{8} \mathrm{GeV} \kappa_{\mathrm{f}}^{-1}\left(\widetilde{m}_{1}\right)
$$

with the last inequality indicating the $3 \sigma$ lower bound. For values of $M_{1} \ll 10^{14} \mathrm{GeV}$ [10] one can use the results of this section for the efficiency factor, neglecting the $\Delta L=2$ washout term. Eq. (119) then provides a lower bound on $M_{1}$ which depends only on $\widetilde{m}_{1}$. 


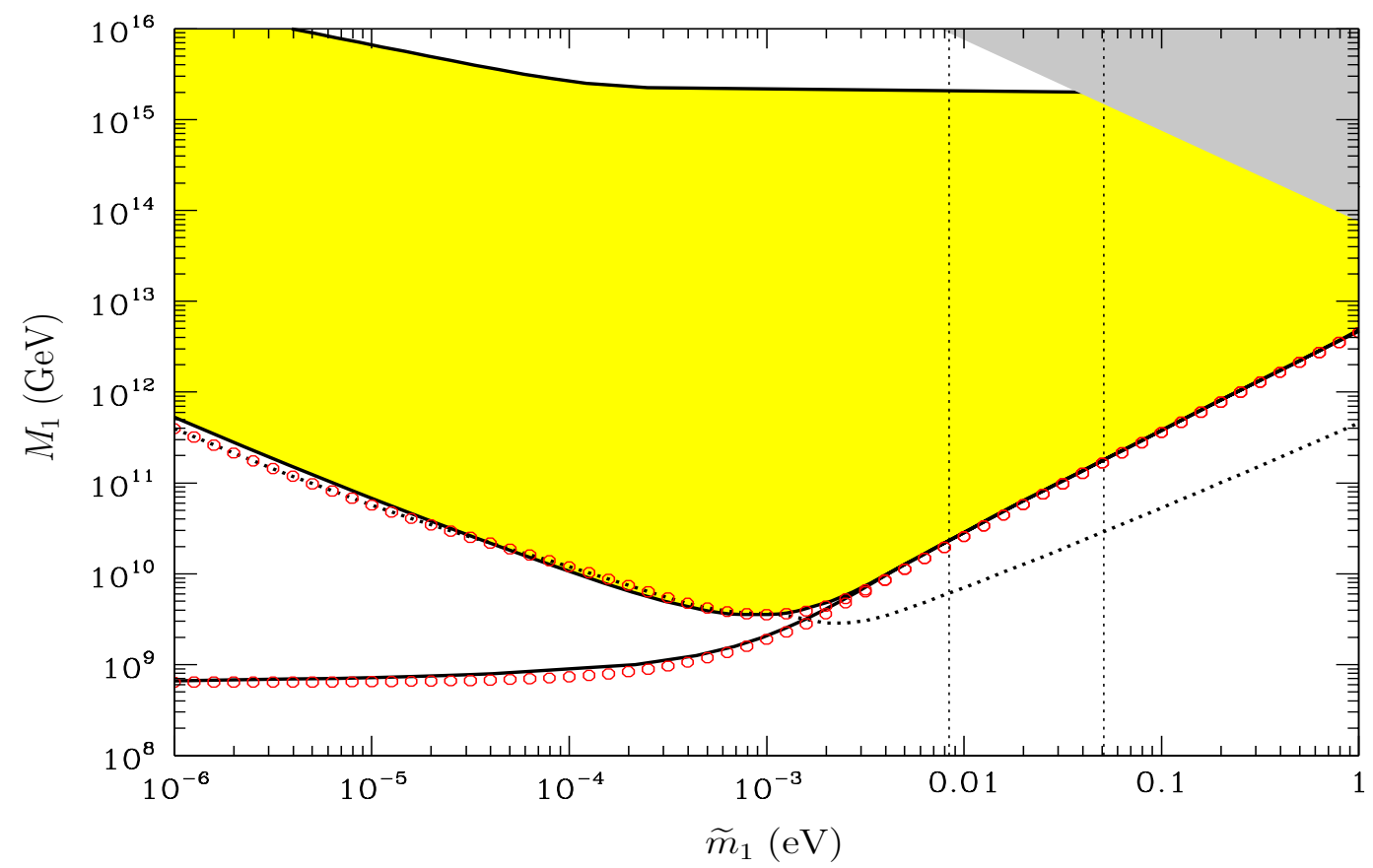

Figure 10: Analytical lower bounds on $M_{1}$ (circles) and $T_{\mathrm{i}}$ (dotted line) for $m_{1}=0$, $\eta_{B}^{C M B}=6 \times 10^{-10}$ and $m_{\text {atm }}=0.05 \mathrm{eV}$. The analytical results are compared with the numerical ones (solid lines). The vertical dashed lines indicate the range $\left(m_{\mathrm{sol}}, m_{\mathrm{atm}}\right)$. The gray triangle at large $M_{1}$ and large $\widetilde{m}_{1}$ is excluded by theoretical consistency (cf. appendix A).

Fig. 10 shows the analytical results for $M_{1}^{\min }\left(\widetilde{m}_{1}\right)$, based on Eq. (107) for thermal initial abundance (thin lines) and the sum of Eqs. (109) and (110) for zero initial abundance (thick lines). For comparison also the numerical results (solid lines) are shown. The absolute minimum for $M_{1}$ is obtained for thermal initial abundance in the limit $\widetilde{m}_{1} \rightarrow 0$, for which $\kappa_{\mathrm{f}}=1$. The corresponding lower bound on $M_{1}$ can be read off from Eq. (120) and at $3 \sigma$ one finds

$$
M_{1} \gtrsim 4 \times 10^{8} \mathrm{GeV} .
$$

This result is in agreement with [10] and also with the recent calculation [12]. Note that the lower bound on $M_{1}$ becomes much more stringent in the case of only two heavy Majorana neutrinos [28]. The bound for thermal initial abundance is model independent. However, it relies on some unspecified mechanism which thermalizes the heavy neutrinos $N_{1}$ before the temperature drops considerably below $M_{1}$. Further, the case $\widetilde{m}_{1} \ll 10^{-3} \mathrm{eV}$ is rather artificial within neutrino mass models, and in this regime a pre-existing asymmetry would not be washed out [2]. 
For zero initial abundance the lower bound is obtained for $\kappa_{0}\left(\widetilde{m}_{1}\right)=\kappa_{\text {peak }} \simeq 0.18$, corresponding to $\widetilde{m}_{1}^{\text {peak }} \simeq 10^{-3} \mathrm{eV}$. In this case one obtains from Eq. (120) [10],

$$
M_{1} \gtrsim 2 \times 10^{9} \mathrm{GeV}
$$

Particularly interesting is the lower bound on $M_{1}$ in the favored neutrino mass range $m_{\text {sol }} \lesssim \widetilde{m}_{1} \lesssim m_{\text {atm }}$. This range lies in the strong washout regime where the simple power law scaling (108) holds. One thus obtains from Eq. (120)

$$
M_{1}^{\min }\left(\widetilde{m}_{1}\right) \simeq(3.3 \pm 0.4) \times 10^{10} \mathrm{GeV}\left(\frac{\widetilde{m}_{1}}{10^{-2} \mathrm{eV}}\right)^{1.1}
$$

which at $3 \sigma$ implies

$$
M_{1} \gtrsim 2 \times 10^{10} \mathrm{GeV}\left(\frac{\widetilde{m}_{1}}{10^{-2} \mathrm{eV}}\right)^{1.1} \simeq\left(10^{10} \div 10^{11}\right) \mathrm{GeV}
$$

where the last range corresponds to values $m_{\text {sol }} \lesssim \widetilde{m}_{1} \lesssim m_{\text {atm }}$. Note that these bounds are fully consistent with neglecting the $\Delta L=2$ washout term, which is justified for $M_{1}^{\min } \ll 10^{14} \mathrm{GeV}$.

In the case of near mass degeneracy between the lightest and the next-to-lightest heavy neutrino $N_{2}, y=\left(M_{2}-M_{1}\right) / M_{1} \ll 1$, the upper bound on the $C P$ asymmetry $\varepsilon_{1}$ is enhanced by a factor $\xi(y) \simeq 1 /(3 y)[29,30]$. The $C P$ asymmetry of $N_{2}$ can also be maximal. Since the number of decaying neutrinos is essentially doubled, one obtains for the reduced lower bound on $M_{1}(y<1)$,

$$
M_{1}>M_{1}^{\min }\left(\widetilde{m}_{1}, y\right)=\frac{M_{1}^{\min }\left(\widetilde{m}_{1}\right)}{2 \xi(y)},
$$

where $M_{1}^{\text {min }}$ is given by Eq. (119). It has been suggested that for extreme degeneracies a resonant regime [11] is reached where $\varepsilon_{1}^{\max }=\mathcal{O}(1)$. In this case there is practically no lower bound on $M_{1}$ from leptogenesis.

\subsection{Lower bound on $T_{\mathrm{i}}$}

It is usually assumed that the lower bound on the initial temperature $T_{\mathrm{i}}$ roughly coincides with $M_{1}^{\text {min }}$, the lower bound on the heavy neutrino mass $M_{1}$. Here $T_{\mathrm{i}}$ can be thought of as the temperature after reheating, below which the universe is radiation dominated [31]. However, in the following we will show that this is only the case in the weak washout regime, i.e. for $\widetilde{m}_{1} \lesssim m_{*} \simeq 10^{-3} \mathrm{eV}$, whereas in the more interesting strong washout regime $T_{\mathrm{i}}$ can be about one order of magnitude smaller than $M_{1}$. 


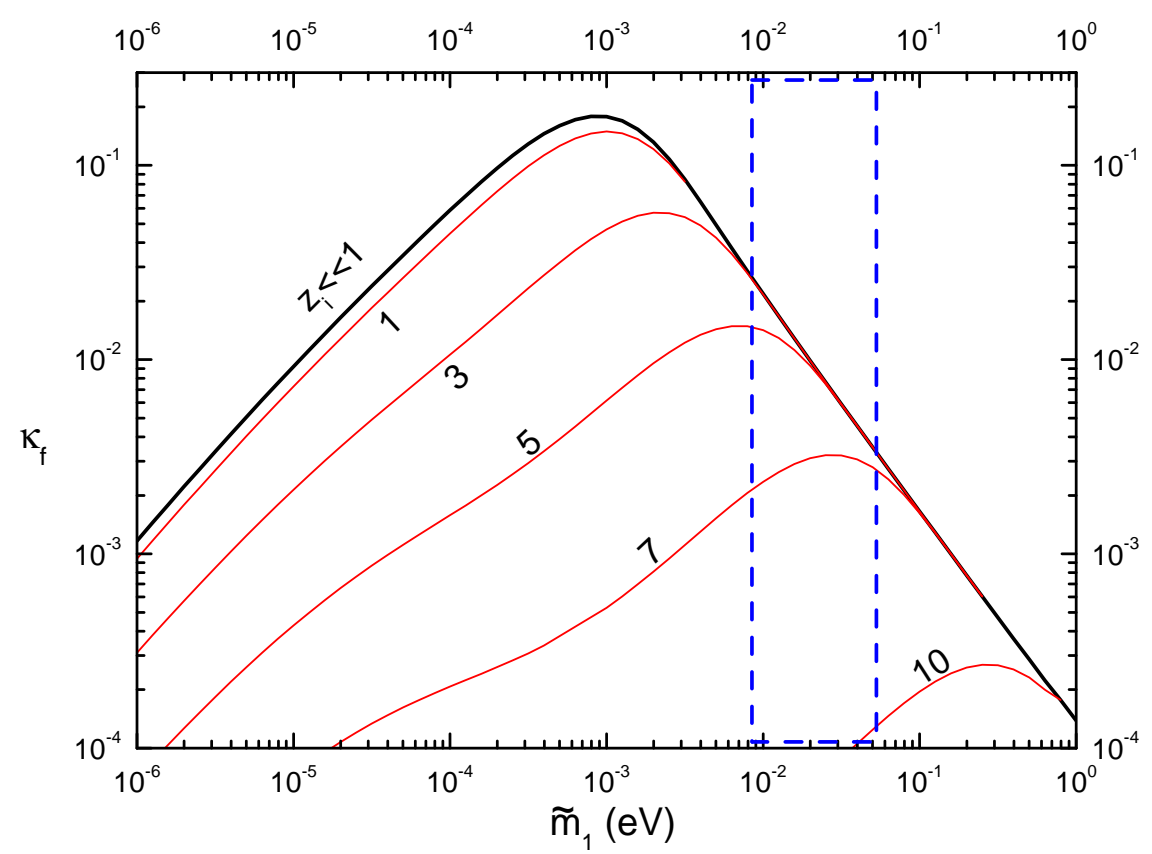

Figure 11: The final efficiency factor for different values of $z_{\mathrm{i}}=M_{1} / T_{\mathrm{i}}$ as indicated. For $z_{\mathrm{i}} \gtrsim z_{B}$ there is a significant suppression.

In general, the maximal baryon asymmetry is a function of both, $M_{1}$ and $T_{\mathrm{i}}=M_{1} / z_{\mathrm{i}}$,

$$
\eta_{B}^{\max }\left(M_{1}, \widetilde{m}_{1}, \bar{m}, z_{\mathrm{i}}\right)=d \varepsilon_{1}^{\max }\left(M_{1}, \widetilde{m}_{1}, \bar{m}\right) \kappa_{\mathrm{f}}\left(\widetilde{m}_{1}, z_{\mathrm{i}}\right) .
$$

In a rigorous procedure one would have to treat $M_{1}$ and $T_{\mathrm{i}}$ as independent variables and to determine the values $z_{\mathrm{i}}^{\max }=M_{1} / T_{\mathrm{i}}^{\min }$ as well as $M_{1}^{\min }$ for which the CMB constraint $\eta_{B}^{\min } \geq \eta_{B}^{C M B}$ is satisfied. This will yield a value $\left.M_{1}^{\min }\right|_{z_{\mathrm{i}}}$ somewhat larger than $\left.M_{1}^{\min }\right|_{z_{\mathrm{i}}=0}$. For simplicity, we shall use the approximation $\left.\left.M_{1}^{\min }\right|_{z_{\mathrm{i}}} \simeq M_{1}^{\min }\right|_{z_{\mathrm{i}}=0}$ in the following. We then define the value $z_{\mathrm{i}}^{\max }$, and the corresponding temperature $T_{\mathrm{i}}^{\min }=M_{1}^{\min } / z_{\mathrm{i}}^{\max }$, by requiring that the final asymmetry $\eta_{B}^{\max }$ agrees with observation within $1 \sigma$ relative error of the quantity $\eta_{B}^{C M B} / m_{\text {atm }}$ which controls $M_{1}^{\min }$.

In the weak washout regime, i.e. $\widetilde{m}_{1}<m_{*}$, one has $z_{\mathrm{i}}^{\max } \simeq 1$. At temperatures smaller than $M_{1}$, the predicted asymmetry rapidly decreases. Either, there is not enough time to produce neutrinos (for zero initial abundance) or the thermal abundance is Boltzmann suppressed (for thermal initial abundance). As Fig. 11 illustrates, for $\widetilde{m}_{1}<m_{*}$ the final efficiency factors for $z_{\mathrm{i}}=1$ and $z_{\mathrm{i}} \ll 1$ differ by only $10 \%$. Hence, in the weak washout regime one has $z_{\mathrm{i}}^{\max } \simeq z_{\mathrm{B}} \simeq 1$.

In the strong washout regime the baryon asymmetry is predominantly produced around $z_{\mathrm{B}}$. The value of $z_{\mathrm{i}}^{\max }$ is thus given by $z_{\mathrm{i}}^{\max } \simeq z_{\mathrm{B}}-1.3 \sigma_{\psi}$, where $\sigma_{\psi}$ is the 


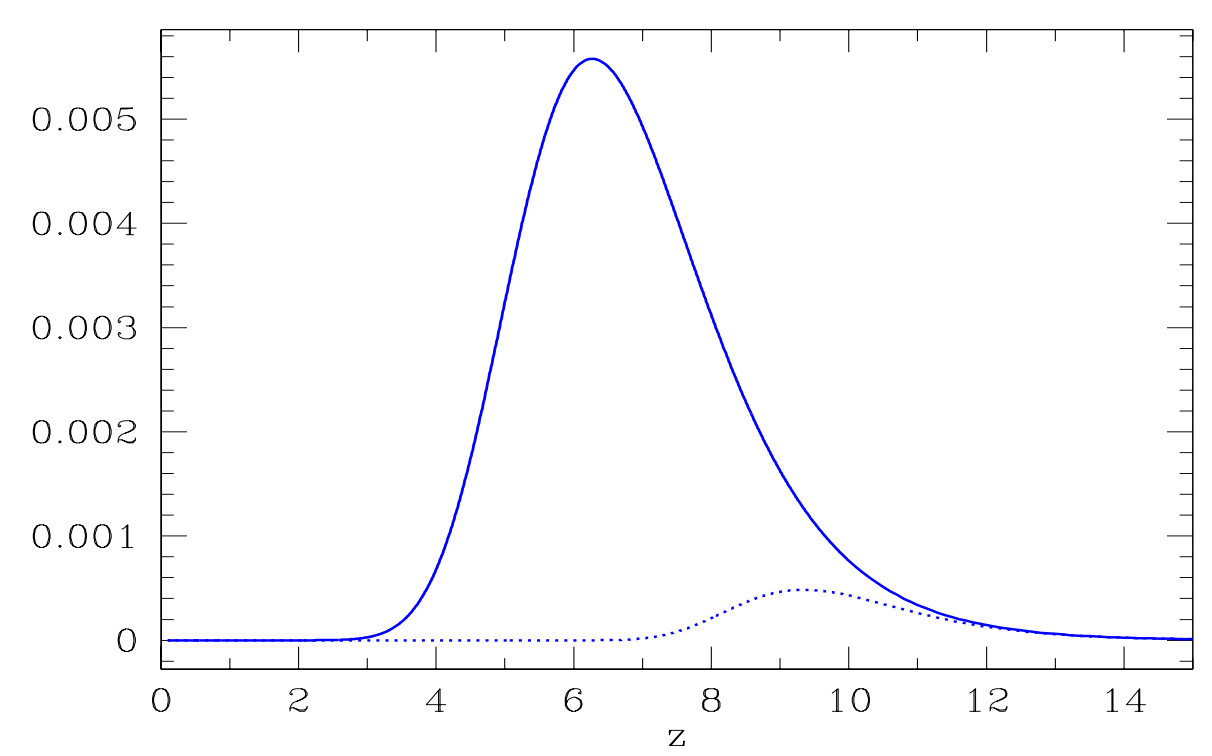

Figure 12: The function $\exp \left(-\psi_{S}(z, \infty)\right)$ (cf. Eq. (106)) for $\widetilde{m}_{1}=10^{-2} \mathrm{eV}$ (full line) and $\widetilde{m}_{1}=10^{-1} \mathrm{eV}$ (dashed line).

width of the Gaussian which approximates the function $\exp \left(-\psi_{S}^{\mathrm{f}}(z)\right)$ (cf. (106)) peaked at $z=z_{\mathrm{B}}$ and whose integral between $z_{\mathrm{i}}^{\max }$ and infinity gives the final efficiency factor minus the small error that is tolerated. In Fig. 12 the function $\exp \left(-\psi_{S}^{\mathrm{f}}(z)\right)$ is shown for $\widetilde{m}_{1}=10^{-2} \mathrm{eV}$ and $\widetilde{m}_{1}=10^{-1} \mathrm{eV}$, respectively. The width of the peak is given by $\sigma_{\psi} \simeq\left(\psi^{\prime \prime}\left(z_{B}\right)\right)^{-1 / 2} \simeq 1.5$ for $\widetilde{m}_{1} \gg m_{*}$.

One can easily write down an approximate expression for $z_{\mathrm{i}}^{\max }(K)$ which interpolates between the two regimes of weak and strong washout,

$$
z_{\mathrm{i}}^{\max }(K) \simeq z_{B}-2 e^{-3 / K} .
$$

The importance of the quantity $z_{B}$ becomes apparent by comparing Figs. 5 and 11. For instance, for $\widetilde{m}_{1}=m_{\mathrm{atm}} \simeq 0.05 \mathrm{eV}$ one has $z_{B} \simeq 8$ and thus $z_{\mathrm{i}}^{\max } \simeq 6$, while for $\widetilde{m}_{1}=$ $m_{\text {sol }} \simeq 0.008 \mathrm{eV}$ one has $z_{B} \simeq 6$ and thus $z_{\mathrm{i}}^{\max } \simeq 4$. Clearly, for values $z_{\mathrm{i}}>z_{\mathrm{i}}^{\max }(K)$ the suppression of the efficiency factor becomes significant.

From Eq. (127) one immediately obtains a lower bound on the initial temperature $T_{\mathrm{i}}$,

$$
T_{\mathrm{i}}>\frac{M_{1}^{\min }}{z_{\mathrm{i}}^{\max }} \simeq \frac{M_{1}^{\min }}{z_{B}-2 e^{-3 / K}} .
$$

The result is shown in Fig. 10. For small $\widetilde{m}_{1} \lesssim m_{*}$ one has $z_{\mathrm{i}}^{\max } \simeq 1$ and consequently $T_{\mathrm{i}}^{\min } \simeq M_{1}^{\min }$. Hence, in particular, the $3 \sigma$ bounds (121) and (122) apply also to $T_{\mathrm{i}}$. More interestingly, in the favored region $m_{\text {sol }} \lesssim \widetilde{m}_{1} \lesssim m_{\text {atm }}$ (dashed box) the $3 \sigma$ bound (124) 
gets relaxed by a factor 4 to 6 and thus

$$
T_{\mathrm{i}}^{\min }=\left(4 \times 10^{9} \div 2 \times 10^{10}\right) \mathrm{GeV}
$$

Therefore, in the favored region of $\widetilde{m}_{1}$ (dashed box), $T_{\mathrm{i}}^{\min }\left(\widetilde{m}_{1}\right)$ is only one order of magnitude higher than the absolute minimum $T_{\mathrm{i}} \simeq 3 \times 10^{9} \mathrm{GeV}$ at $\widetilde{m}_{1} \simeq m_{*}$ (zero initial abundance) and less than two orders of magnitude higher than the asymptotical minimum for $\widetilde{m}_{1} \ll m_{*}$ (thermal initial abundance). This is important in view of the 'gravitino problem' which yields an upper bound on $T_{\mathrm{i}}$ for some supersymmetric extensions of the standard model.

Comparing our results with those of [12], where the additional $B-L$ asymmetry has been calculated which is produced during the reheating period at temperatures above $T_{\text {reh }}$ and below some maximal temperature $T_{\max }$, we find the same amount of relaxation of the bound on $T_{\mathrm{i}}=T_{\mathrm{reh}}$. This indicates that the relaxation is a consequence of $T_{B}<M_{1}^{\text {min }}$ in the case of strong washout rather than the existence of a non radiation dominated regime above $T_{\text {reh. }}$.

\section{Upper bound on the light neutrino masses}

We now want to study the effect of the contribution $\Delta W$ to the total washout. This term originates from the $\Delta L=2$ processes $\phi l \leftrightarrow \bar{\phi} \bar{l}$ and $\phi \phi \leftrightarrow \overline{l l}$ with the heavy neutrinos $N_{1}$, $N_{2}$ and $N_{3}$ in s- and t-channel, respectively. $\Delta W$ is the only term in the kinetic equations which is not proportional to $\widetilde{m}_{1}$ but instead to the heavy neutrino mass $M_{1}$.

At low temperatures the washout term $\Delta W$ reads,

$$
\Delta W(z) \simeq \frac{\omega}{z^{2}}\left(\frac{M_{1}}{10^{10} \mathrm{GeV}}\right)\left(\frac{\bar{m}}{\mathrm{eV}}\right)^{2},
$$

where $\bar{m}$ is the absolute neutrino mass scale, and the dimensionless constant $\omega$ is given by

$$
\omega=\frac{9 \sqrt{5} M_{p} 10^{-8} \mathrm{GeV}^{3}}{4 \pi^{9 / 2} g_{l} \sqrt{g_{\star}} v^{4}} \simeq 0.186 .
$$

$\Delta W$ is compared in Fig. 13 of appendix $\mathrm{A}$ with the total washout term

$$
W(z)=W_{0}\left(z ; \widetilde{m}_{1}\right)+\Delta W\left(z ; M_{1} \bar{m}^{2}\right) .
$$

As discussed in the appendix, there is a sharp transition to a low temperature regime where $\Delta W$ dominates over $W_{0}$. This transition occurs for a value $z_{\Delta} \gg 1$, which is determined by

$$
W_{0}\left(z_{\Delta}\right)=\Delta W\left(z_{\Delta}\right) .
$$


From Eqs. (105) and (130) one easily obtains,

$$
K z_{\Delta}^{9 / 2} e^{-z_{\Delta}} \sim \omega\left(\frac{M_{1}}{10^{10} \mathrm{GeV}}\right)\left(\frac{\bar{m}}{\mathrm{eV}}\right)^{2} .
$$

In the case $z_{\Delta} \gtrsim z_{\mathrm{B}}$, the values of $z_{\mathrm{B}}$ and of the efficiency factor at $z \sim z_{\Delta}$ are not affected by $\Delta W$. Since for $z>z_{\mathrm{B}}$ no asymmetry is produced, the total efficiency factor is simply given by

$$
\bar{\kappa}_{\mathrm{f}}\left(\widetilde{m}_{1}, M_{1} \bar{m}^{2}\right)=\kappa_{\mathrm{f}}\left(\widetilde{m}_{1}\right) e^{-\int_{z_{\mathrm{B}}}^{\infty} d z \Delta W(z)},
$$

where the second factor describes the modification due to the presence of $\Delta W$. Note that $\bar{\kappa}_{\mathrm{f}}$ depends on $\widetilde{m}_{1}$ also via $z_{\mathrm{B}}$. For $z_{B} \gtrsim 3$ we can use the low temperature limit (130) for $\Delta W$, which yields

$$
\bar{\kappa}_{\mathrm{f}}\left(\widetilde{m}_{1}, M_{1} \bar{m}^{2}\right)=\kappa_{\mathrm{f}}\left(\widetilde{m}_{1}\right) e^{-\frac{\omega}{z^{2}}\left(\frac{M_{1}}{10^{10} \mathrm{GeV}}\right)\left(\frac{\bar{m}}{\mathrm{eV}}\right)^{2}} .
$$

Given the solar and atmospheric mass squared differences and a neutrino mass pattern, i.e. $m_{3}^{2}-m_{2}^{2}>m_{2}^{2}-m_{1}^{2}$ or $m_{3}^{2}-m_{2}^{2}<m_{2}^{2}-m_{1}^{2}$, the dependence of $m_{3}$ on $m_{1}$ is fixed. In Ref. [2] the absolute neutrino mass scale $\bar{m}$ was used as variable. In the following we prefer to use instead the lightest neutrino mass $m_{1}$. In the case of normal hierarchy, with $m_{3}^{2}-m_{2}^{2}=\Delta m_{\text {atm }}^{2}$ and $m_{2}^{2}-m_{1}^{2}=\Delta m_{\text {sol }}^{2}$, one has

$$
\begin{aligned}
& m_{3}^{2}=m_{1}^{2}+\Delta m_{\mathrm{atm}}^{2}+\Delta m_{\mathrm{sol}}^{2} \\
& m_{2}^{2}=m_{1}^{2}+\Delta m_{\mathrm{sol}}^{2} \\
& \bar{m}^{2}=3 m_{1}^{2}+\Delta m_{\mathrm{atm}}^{2}+2 \Delta m_{\mathrm{sol}}^{2}
\end{aligned}
$$

In the case of inverted hierarchy analogous relations hold.

Consider now the maximal baryon asymmetry (cf. (10)),

$$
\eta_{B}^{\max }\left(\widetilde{m}_{1}, M_{1}, m_{1}\right) \simeq d \varepsilon_{1}^{\max }\left(\widetilde{m}_{1}, M_{1}, m_{1}\right) \bar{\kappa}_{\mathrm{f}}\left(\widetilde{m}_{1}, M_{1}, m_{1}\right)
$$

In the case $m_{1}=0$ the maximal $C P$ asymmetry was depending only on $M_{1}$ (cf. Eq. (118)). If $m_{1} \geq 0$ this is suppressed by a function $\beta\left(\widetilde{m}_{1}, m_{1}\right) \leq 1$ depending both on $m_{1}$ and on $\widetilde{m}_{1}[24,2]$ such that

$$
\varepsilon_{1}^{\max }\left(M_{1}, \widetilde{m}_{1}, m_{1}\right)=\varepsilon_{1}^{\max }\left(M_{1}\right) \beta\left(\widetilde{m}_{1}, m_{1}\right)
$$

The maximal value $\beta=1$ is obtained in the case $m_{1}=0$. The function $\beta$ is conveniently factorized,

$$
\beta\left(\widetilde{m}_{1}, m_{1}\right)=\beta_{\max }\left(m_{1}\right) f\left(\widetilde{m}_{1}, m_{1}\right)
$$


The first factor,

$$
\beta_{\max }\left(m_{1}\right)=\frac{\left(m_{3}-m_{1}\right)}{m_{\mathrm{atm}}}=\frac{m_{\mathrm{atm}}}{\left(m_{3}+m_{1}\right)},
$$

is the maximal value of $\beta$ for fixed $m_{1}$, which decreases $\propto 1 / m_{1}$ for $m_{1} \gg m_{\text {atm }}$. The function $f$ contains the dependence on $\widetilde{m}_{1}$,

$$
f\left(\widetilde{m}_{1}, m_{1}\right) \simeq \frac{\left(m_{3}-m_{1} \sqrt{1+\frac{m_{\mathrm{atm}}^{2}}{\widetilde{m}_{1}^{2}}}\right)}{m_{3}-m_{1}} .
$$

This expression describes correctly the behavior of the maximal $C P$ asymmetry in the limits $m_{1} \rightarrow 0$ and $\widetilde{m}_{1} \rightarrow \infty$. However, it has recently been pointed out [32] that Eq. (144) underestimates the maximal $C P$ asymmetry in particular in the regime of quasidegenerate neutrinos ${ }^{4}$. For simplicity, we shall first calculate the neutrino mass bound using Eq. (144) and then discuss the correction.

Let us now calculate the value $M_{1}$ that maximizes $\eta_{B}^{\max }$. In the $\left(\widetilde{m}_{1}, M_{1}\right)$-plane this defines a trajectory $\eta_{B}^{\max }\left(\widetilde{m}_{1}, m_{1}\right)$ along which $\eta_{B}^{\max }$ is maximal with respect to $M_{1}$. The corresponding condition,

$$
\frac{d \ln \eta_{B}^{\max }}{d M_{1}}=0
$$

yields the relation,

$$
\frac{\omega}{z_{B}}\left(\frac{M_{1}}{10^{10} \mathrm{GeV}}\right)\left(\frac{\bar{m}}{\mathrm{eV}}\right)^{2}=1,
$$

where the quantity $z_{B}$ is a function of $\tilde{m}_{1}$. It is now easy to see that the ratio $\eta_{B}^{\max } / \eta_{B}^{C M B}$, maximized with respect to $M_{1}$, can be expressed in the following form,

$$
\frac{\eta_{B}^{\max }\left(\widetilde{m}_{1} ; m_{1}\right)}{\eta_{B}^{C M B}} \simeq \chi \xi \frac{z_{\mathrm{B}}\left(\widetilde{m}_{1}\right) f\left(\widetilde{m}_{1}, m_{1}\right) \kappa_{\mathrm{f}}\left(\widetilde{m}_{1}\right)}{\left(m_{1}+m_{3}\right) \bar{m}^{2}}
$$

where $\chi$ is the constant

$$
\chi=\frac{25 d}{6 e \omega} \frac{\mathrm{eV}^{4}}{m_{0}} \simeq 1.6 \mathrm{eV}^{3} .
$$

and $m_{0}=(16 \pi / 3)\left(v^{2} / 10^{16} \mathrm{GeV}\right) \simeq 0.051 \mathrm{eV}$. The parameter $\xi$ is the product

$$
\xi=\frac{\xi_{\varepsilon} \xi_{\mathrm{atm}}^{2} \xi_{0}}{\xi_{\eta} \xi_{\Delta}} .
$$

\footnotetext{
${ }^{4}$ The expression (144) has been obtained using Eq. (22) in Ref. [2] and assuming $x_{3}=0$, which is valid only in the limit $m_{1} \rightarrow 0$. Note, however, that Eq. (144) approximates the maximal $C P$ asymmetry within about $20 \%$ also in the quasi-degenerate case for the relevant values of $\widetilde{m}_{1}$. For quasi-degenerate neutrinos, with $m_{1} \simeq m_{3}<\widetilde{m}_{1}$, one easily finds that the maximal $C P$ asymmetry is reached for $x_{3} \simeq m_{1} /\left(2 \widetilde{m}_{1}\right)$.
} 
It accounts for various factors affecting the ratio $\eta_{B}^{\max } / \eta_{B}^{C M B}:(1)$ the maximal $C P$ asymmetry, $\xi_{\varepsilon} ;(2)$ the atmospheric neutrino mass scale, $\xi_{\text {atm }}=m_{\text {atm }} /(0.05 \mathrm{eV})$; (3) the observed baryon asymmetry, $\xi_{\eta}=\eta_{B}^{C M B} /\left(6 \times 10^{-10}\right)$; (4) the variation $\xi_{\Delta}$ of the strength of the $\Delta L=2$ washout term, and (5) the variation $\xi_{0}$ of the efficiency factor at small $M_{1} \bar{m}^{2}$. This parametrization of the maximal asymmetry is useful to study the dependence of the neutrino mass bound on the various parameters involved.

In order to determine the absolute maximum of the asymmetry $\eta_{B}^{\max }$ we also have to find the extremum with respect to $\widetilde{m}_{1}$ and, finally, the maximum with respect to $m_{1}$ or, equivalently, the absolute neutrino mass scale. Comparison with the observed asymmetry $\eta_{B}^{C M B}$ then yields the leptogenesis neutrino mass bound. Anticipating again that the maximum falls in the region of large $\widetilde{m}_{1}$, we can use the analytical expression (107) for $\kappa_{\mathrm{f}}$ in the strong washout region. Since $j\left(z_{B} \gg 1\right) \simeq 1$, one has

$$
z_{\mathrm{B}}\left(\widetilde{m}_{1}\right) \kappa_{\mathrm{f}}\left(\widetilde{m}_{1}\right) \simeq \frac{2}{K}=\frac{2 m_{*}}{\widetilde{m}_{1}}
$$

Further, for large $\widetilde{m}_{1}$ the function $f\left(\widetilde{m}_{1}, m_{1}\right)$ can be approximated by

$$
f\left(\widetilde{m}_{1}, m_{1}\right) \simeq 1-\frac{1}{2} \frac{\left(m_{3}+m_{1}\right) m_{1}}{\widetilde{m}_{1}^{2}} .
$$

With this simplified expression it is easy to see that the peak is reached for

$$
\widetilde{m}_{1}^{\text {peak }} \simeq \sqrt{\frac{3}{2} m_{1}\left(m_{1}+m_{3}\right)}
$$

corresponding to $f\left(\widetilde{m}_{1}^{\text {peak }}, m_{1}\right)=2 / 3$. The peak value of the asymmetry is given by

$$
\frac{\eta_{B}^{\text {peak }}\left(m_{1}\right)}{\eta_{B}^{C M B}} \simeq \frac{2^{5 / 2}}{3^{3 / 2}} \frac{\chi m_{*} \xi}{\left(m_{1}+m_{3}\right)^{3 / 2} m_{1}^{1 / 2} \bar{m}^{2}}
$$

Anticipating $\left(m_{1}^{\text {peak }}\right)^{2} \gg m_{\text {atm }}^{2}$, one has to zeroth order in $\left(m_{\text {atm }} / m_{1}\right)^{2}$,

$$
m_{3}^{0}=\frac{\bar{m}^{0}}{\sqrt{3}} \simeq m_{1}
$$

Imposing now the CMB constraint $\eta_{B} \geq \eta_{B}^{C M B}$ we find the leptogenesis bound on the absolute neutrino mass scale (cf. [33, 2]),

$$
m_{i} \leq m_{\mathrm{peak}}^{0}=\sigma \xi^{1 / 4} \mathrm{eV}
$$

with

$$
\sigma=10^{6}\left(\frac{10 g_{\mathrm{rec}} a_{\mathrm{sph}} \pi^{6}}{3^{9 / 2} e}\right)^{1 / 4}\left(\frac{v^{2}}{M_{\mathrm{PL}} \mathrm{GeV}}\right)^{1 / 2} \simeq 0.121
$$


In this last equation we used the fact that in a standard thermal history the dilution factor, contained in $d$, is given by $N_{\gamma}^{\mathrm{rec}} / N_{\gamma}^{\star}=g_{*} / g_{\mathrm{rec}}$ with $g_{\mathrm{rec}}=43 / 11$ the number of the (entropy) degrees of freedom at recombination. Combining Eqs. (154) and (152) one finds

$$
\widetilde{m}_{1}^{\text {peak }} \simeq \bar{m}_{\text {peak }}^{0} \simeq 0.17 \mathrm{eV}
$$

which is consistent with the approximation of strong washout used in Eq. (150). From Fig. 5 one then reads off $z_{B}^{\text {peak }} \simeq 10$. Together with (146) this yields for the peak value of $M_{1}$,

$$
M_{1}^{\text {peak }}=\frac{z_{\mathrm{B}}\left(\widetilde{m}_{1}^{\text {peak }}\right)}{\omega\left(\bar{m}_{\text {peak }}^{0}\right)^{2}} \simeq 2 \times 10^{13} \xi^{-1 / 2} \mathrm{GeV} .
$$

It is straightforward to go beyond the zeroth order in $\left(m_{\mathrm{atm}} / m_{1}\right)^{2}$. In the case of normal hierarchy the lightest neutrino mass bound is given by

$$
m_{1, \text { nor }}^{\text {peak }} \simeq m_{\text {peak }}^{0}\left(1-\frac{17}{96} \frac{m_{\text {atm }}^{2}}{\left(m_{\text {peak }}^{0}\right)^{2}}\right),
$$

whereas in the case of inverted hierarchy one has

$$
m_{1, \text { inv }}^{\text {peak }} \simeq m_{\text {peak }}^{0}\left(1-\frac{25}{96} \frac{m_{\mathrm{atm}}^{2}}{\left(m_{\text {peak }}^{0}\right)^{2}}\right),
$$

which yields $m_{1, \text { nor }}^{\text {peak }} \simeq m_{1, \text { inv }}^{\text {peak }} \simeq m_{\text {peak }}^{0}-0.005 \mathrm{eV}$. In order to obtain numerical results for the upper bounds on the light neutrino masses one has to specify the baryon asymmetry and the neutrino mass squared differences. For $\eta_{B}^{C M B}$ we use the WMAP plus SDSS result (111), while the value for $m_{\text {atm }}$ is given by the Eq. (117). Since $\bar{m}_{\text {peak }} \propto\left(m_{\text {atm }}^{2} / \eta_{C M B}\right)^{1 / 4}$, the experimental error on $m_{\text {peak }}^{0}$ is about $5 \%$. Setting all other parameters $\xi_{i}=1$, one finds for the central value $\xi=\xi_{\text {atm }}^{2} / \xi_{\eta} \simeq 0.95$. We then obtain

$$
m_{1}^{\text {peak }}=(0.115 \pm 0.005) \mathrm{eV} .
$$

The corresponding $3 \sigma$ upper bounds on the neutrino masses are for normal hierarchy,

$$
m_{1}, m_{2}<0.13 \mathrm{eV}, \quad m_{3}<0.14 \mathrm{eV},
$$

and correspondingly in the case of inverted hierarchy,

$$
m_{1}<0.13 \mathrm{eV}, \quad m_{2}, m_{3}<0.14 \mathrm{eV} \text {. }
$$

These analytical bounds are consistent with the numerical results obtained in [2], if one accounts for the different parameters, $m_{\text {atm }}=0.05 \mathrm{eV}$ and $\eta_{C M B}=3.6 \times 10^{-10}(\xi \simeq 1.7)$, and the over-estimate of the washout term $W_{0}$ by $50 \%$. 
The bound on the maximal $C P$ asymmetry derived in [32] corresponds in the relevant range of large $m_{1}$ and $\widetilde{m}_{1}$ to the function (cf. (144)),

$$
f\left(\widetilde{m}_{1}, m_{1}\right) \simeq \sqrt{1-\frac{m_{1}^{2}}{\widetilde{m}_{1}^{2}}} .
$$

Repeating the above analysis one finds that the peak of the asymmetry is shifted to $\widetilde{m}_{1}^{\text {peak }}=\sqrt{2} m_{1}$, with $f^{\text {peak }} \simeq 1 / \sqrt{2}$. From Eqs. $(150),(154)$ and (157) one then concludes that the neutrino mass bound is relaxed by the factor $\left(3^{3 / 2} / 4\right)^{1 / 4} \simeq 1.07$, i.e. $7 \%$, corresponding to an increase of the neutrino mass bound by $0.01 \mathrm{eV}$.

An important correction arises from the dependence of the neutrino masses on the renormalization scale $\mu$. The only low energy quantity upon which $m_{1}^{\text {peak }}$ depends is the atmospheric neutrino mass scale $m_{\mathrm{atm}}$. Hence, there are two competing effects: the first one is the running of $m_{\text {atm }}$ from the Fermi scale $\mu=m_{Z}$ to the high scale $\mu \sim M_{1}$ $\left(\sim 10^{13} \mathrm{GeV}\right)$, the second one is the running of $m_{1}^{\text {peak }}$ from $\mu \sim M_{1}$ down to $\mu=m_{Z}$. In the standard model the light neutrino masses scale uniformly under the renormalization group. Since $m_{\text {peak }} \propto \sqrt{m_{\text {atm }}}$, the first effect then gives a correction that is only half of the second one. Renormalization group effects make the bound more restrictive [34]. In order to have an upper bound, we have to choose those values of the parameters that produce the smallest effect. This corresponds to choosing the lowest Higgs mass compatible with positive Higgs self-coupling at $\sim 10^{13} \mathrm{GeV}$, which is about $150 \mathrm{GeV}$. The atmospheric neutrino mass scale is then increased by about $40 \%$ [34] and the bound gets $20 \%$ weaker at $\mu \sim M_{1}$, but $20 \%$ more restrictive at $\mu=m_{Z}$. Combining the effect of radiative corrections and the larger $C P$ asymmetry (Eq. (164)), we finally obtain from Eq. (162) and (163) at $3 \sigma$,

$$
m_{i}<0.12 \mathrm{eV},
$$

which, thanks to cancellations among different corrections, agrees with the bound obtained in $[2]$.

It is important to realize, however, that there are corrections of the same order as those discussed above which cannot be treated within the present framework. It is usually assumed that in leptogenesis first a lepton asymmetry is produced, which is then partially transformed into a baryon asymmetry by sphaleron processes. However, this picture is incorrect [35]. The duration of leptogenesis is about two orders of magnitude larger than the inverse Hubble parameter when it starts. Since many processes in the plasma, in particular the sphaleron processes, are much faster, the generated asymmetry is 'instantaneously' distributed among quarks and leptons. Hence, the chemical potentials of quarks and leptons are changed already during the process of leptogenesis. A complete 
analysis has to take into account how the contributing 'spectator processes', which are in thermal equilibrium, change with decreasing temperature (cf., e.g., [36]). In [35] is has been estimated that spectator processes reduce the generated baryon asymmetry by about a factor of two. Hence, there is presently a theoretical uncertainty of at least $0.02 \mathrm{eV}$ on the neutrino mass bound.

Finally, it has to be kept in mind that our whole analysis is based on the simplest version of the seesaw mechanism with hierarchical heavy Majorana neutrinos. The leptogenesis neutrino mass bound can be relaxed if the heavy Majorana neutrinos are, at least partially, quasi-degenerate in mass. In this case the $C P$ asymmetry can be much larger $[29,30]$ than the upper bound used in the above discussion. This possibility has to be discussed in the context of realistic models of neutrino masses. Further, if Higgs triplets contribute significantly to neutrino masses the connection between baryon asymmetry and neutrino masses disappears entirely. Different relations between neutrino masses and baryon asymmetry are also obtained in non-thermal leptogenesis [37, 22].

\section{Towards the theory of thermal leptogenesis}

The goal of leptogenesis is the prediction of the baryon asymmetry, given neutrino masses and mixings. The consistency of present calculations with observations is impressive, but so far it is not possible to quote a rigorous theoretical error on the predicted asymmetry, which is a necessary requirement for a 'theory of leptogenesis'.

The generation of a baryon asymmetry is a non-equilibrium process which is generally treated by means of Boltzmann equations. This procedure has a basic conceptual problem: the Boltzmann equations are classical equations for the time evolution of phase space distribution functions; the involved collision terms, however, are zero temperature Smatrix elements which involve quantum interferences in a crucial manner. Clearly, a full quantum mechanical treatment is necessary to understand the range of validity of the Boltzmann equations and to determine the size of corrections.

A first step in this direction has been made in [38] where a perturbative solution of the exact Kadanoff-Baym equations has been constructed. To zeroth order, for non-relativistic heavy neutrinos, the non-equilibrium Green functions have been obtained in terms of distribution functions satisfying Boltzmann equations. Note that in the favoured strong washout regime the decaying heavy neutrinos are indeed non-relativistic. It is instructive to recall the various corrections. There are off-shell contributions, 'memory effects' related to the derivative expansion of the Wigner transforms, relativistic corrections and higherorder loop corrections. All these correction terms are known explicitly, but their size 
during the process of baryogenesis and, in particular, their effect on the final baryon asymmetry have not yet been worked out.

Recently, thermal corrections have been studied [12]. They correspond to loop corrections involving gauge bosons and the top quark. At large temperatures, $T>M_{1}$, the processes in the plasma and the $C P$ asymmetries change significantly if thermal masses are treated as kinematical masses in the evaluation of scattering matrix elements [12]. On the contrary, thermal corrections are small if they are only included as propagator effects [14]. To clarify this issue is of general importance for the treatment of non-equilibrium processes at high temperatures.

The effect of all these corrections on the final baryon asymmetry depends crucially on the value of the neutrino masses. Large thermal corrections would modify the asymmetry at temperatures above $M_{1}$. This affects the final baryon asymmetry only in the case of small washout, i.e. $\widetilde{m}_{1}<m_{*}$. In the strong washout regime, $\widetilde{m}_{1}>m_{*}$, which appears to be favored by the current evidence for neutrino masses, the baryon asymmetry is generated at a temperature $T_{B}<M_{1}$. In this case thermal corrections are small. Correspondingly, the recently obtained bounds on light and heavy neutrino masses $[2,12,32]$ are all very similar.

The final value of the baryon asymmetry is significantly affected by 'spectator processes' [35] which cannot be treated based on the simple Boltzmann equations discussed in this paper. It has been estimated that this effect changes the baryon asymmetry by a factor of about two, leading to a theoretical uncertainty of the leptogenesis neutrino mass bound of about $0.02 \mathrm{eV}$. Clearly, to obtain a more accurate prediction for the baryon asymmetry requires a considerable increase in the complexity of the calculation.

An important step towards the theory of leptogenesis would be a systematic evaluation of all corrections to the simple Boltzmann equations in the 'easy regime' of strong washout where $\widetilde{m}_{1}>m_{*}$. One could then see where this approach breaks down as $\widetilde{m}_{1}$ decreases and $T_{B}$ approaches $M_{1}$. On the experimental side, information on the absolute neutrino mass scale $\bar{m}$, and therefore on $\widetilde{m}_{1}>m_{1}$, is of crucial importance. Maybe, we are lucky, and nature has chosen neutrino masses in the strong washout regime where leptogenesis works best.

\section{Acknowledgments}

We would like to thank L. Covi, K. Hamaguchi, J. Pati and M. Ratz for helpful discussions, and G. Kane and the Michigan Center for Theoretical Physics (MCTP) for hospitality during the Baryogenesis Workshop. P.D.B. thanks the DESY and CERN theory groups for their kind hospitality. The work of P.D.B. has been supported by the EU Fifth Framework network "Supersymmetry and the Early Universe" (HPRN-CT-2000-00152). 


\section{Appendix A}

A crucial and delicate point in setting up the Boltzmann equations for leptogenesis is the subtraction of the real intermediate state contribution (RIS) from the $2 \rightarrow 2$ scattering processes [4]. Without this subtraction, decays and inverse decays lead to the generation of a lepton asymmetry in thermal equilibrium, in contradiction with general theorems.

In order to explicitly split the $2 \rightarrow 2$ scattering processes into RIS and remainder one has to calculate the $\Delta L=2$ processes, including one-loop self-energy and vertex corrections in the resonance region. This calculation has been carried out in [39] where the relevant results are given in Eqs. (68) - (85).

Let us consider for simplicity the case $s, M_{1}^{2} \ll M_{2}^{2}, M_{3}^{2}(s, t$ and $u$ are the usual Mandelstam variables). For our purposes it is sufficient to study the averaged matrix element squared,

$$
|\mathcal{M}(\bar{l} \bar{\phi} \rightarrow l \phi)|_{a v}^{2}=\int_{-s}^{0} d u|\mathcal{M}(\bar{l} \bar{\phi} \rightarrow l \phi)|^{2},
$$

where the integral over $u$ corresponds to the integral over the final state lepton angle, i.e. a partial phase space integration.

In order to study the resonance region the diagonal part of the self-energy was resummed in [39] whereas the off-diagonal part was treated as perturbation in the Yukawa couplings $h$. In the $2 \rightarrow 2$ scattering amplitude the free propagator is then replaced by a Breit-Wigner propagator,

$$
\frac{1}{s-M_{1}^{2}+i M_{1} \Gamma_{1}}=\frac{1}{M_{1}^{2}}\left(\frac{1}{D_{1}(x)}-i \frac{1}{R_{1}(x)}\right),
$$

where

$$
\frac{1}{D_{1}(x)}=\frac{x-1}{(x-1)^{2}+c^{2}}, \quad \frac{1}{R_{1}(x)}=\frac{c}{(x-1)^{2}+c^{2}}
$$

and

$$
\frac{1}{D_{1}(x)^{2}}+\frac{1}{R_{1}(x)^{2}}=\frac{1}{c} \frac{1}{R_{1}(x)}
$$

with

$$
x=\frac{s}{M_{1}^{2}}, \quad c=\frac{\Gamma_{1}}{M_{1}}=\frac{1}{8 \pi} K_{11}, \quad K_{i j}=\left(h^{\dagger} h\right)_{i j} ;
$$

here $h_{k j}$ is the Yukawa coupling of $N_{j}$ to $l_{k} \phi$. 
The averaged matrix elements are then given by the following expression [39],

$$
\begin{aligned}
|\mathcal{M}(\bar{l} \bar{\phi} \rightarrow l \phi)|_{a v}^{2} & =2 s^{2} \sum_{i j}\left(A_{i j}-B_{i j}-C_{i j}-4 s \sum_{k}\left(D_{i j k}+\bar{E}_{i j k}\right)\right), \\
|\mathcal{M}(l \phi \rightarrow \bar{l} \bar{\phi})|_{a v}^{2} & =2 s^{2} \sum_{i j}\left(A_{i j}+B_{i j}+C_{i j}+4 s \sum_{k}\left(D_{i j k}+\bar{E}_{i j k}\right)\right),
\end{aligned}
$$

where we have only shown terms contributing to the subtraction of the RIS part as well as the leading order off-shell part, e.g. $C P$ conserving one-loop corrections have not been included. $A_{i j}$ and $B_{i j}$ represent the various $N_{i} N_{j}$ s-channel interference terms. Up to terms $\mathcal{O}\left(h^{6}\right)$ they are $(i, j=2,3)$

$$
\begin{aligned}
A_{11} & =K_{11}^{2} \frac{1}{M_{1}^{2}}\left(\frac{1}{D_{1}^{2}}+\frac{1}{R_{1}^{2}}\right), \\
A_{1 i}+A_{i 1} & =-2 \operatorname{Re}\left\{K_{1 i}^{2}\right\} \frac{1}{M_{1} M_{i}} \frac{1}{D_{1}}, \\
A_{i j} & =\operatorname{Re}\left\{K_{i j}^{2}\right\} \frac{1}{M_{i} M_{j}}, \\
B_{11} & =B_{i j}+B_{j i}=0, \\
B_{1 i}+B_{i 1} & =2 \operatorname{Im}\left\{K_{1 i}^{2}\right\} \frac{1}{M_{1} M_{i}} \frac{1}{R_{1}}
\end{aligned}
$$

the $N_{1}-N_{1}$ s-channel terms with self-energy and vertex corrections, respectively, read $(k=2,3)$

$$
\begin{aligned}
C_{1 k} & =-2 x \operatorname{Im}\left\{K_{1 k}^{2}\right\} \frac{c}{M_{1} M_{k}}\left(\frac{1}{D_{1}^{2}}+\frac{1}{R_{1}^{2}}\right), \\
D_{11 k} & =\frac{1}{2} \operatorname{Im}\left\{K_{1 k}^{2}\right\} \frac{c}{\sqrt{x} M_{1}^{4}} f\left(\frac{M_{k}^{2}}{s}\right)\left(\frac{1}{D_{1}^{2}}+\frac{1}{R_{1}^{2}}\right),
\end{aligned}
$$

where

$$
\begin{aligned}
f(y) & =\sqrt{y}\left(1-(1+y) \ln \left(\frac{1+y}{y}\right)\right) \\
& =-\frac{1}{2 \sqrt{y}}+\mathcal{O}\left(\frac{1}{y}\right)
\end{aligned}
$$

finally, the s-u-channel interference term is

$$
\bar{E}_{11 k}=-D_{11 k} .
$$

From these equations one reads off

$$
D_{11 k}+\bar{E}_{11 k}=0,
$$


and, for $s=M_{1}^{2}$,

$$
B_{1 i}+B_{i 1}+C_{1 i}=0 .
$$

Hence, the $C P$ asymmetry of the full $2 \rightarrow 2$ cross section vanishes to $\mathcal{O}\left(h^{4}\right)$. The 'pole terms', corresponding to $N_{1}-N_{1}$ s-channel contributions, are cancelled by on-/off-shell s-channel interferences (self-energy) and s-channel/u-channel interference (vertex correction). Off-shell, the corresponding cancellations take place to $\mathcal{O}\left(h^{6}\right)[40]$.

As an unstable particle the heavy neutrino $N_{1}$ is defined as pole in the $2 \rightarrow 2$ scattering amplitude

$$
\mathcal{M}(\bar{l} \bar{\phi} \rightarrow l \phi) \simeq\left\langle l \phi \mid N_{1}\right\rangle \frac{i}{s-M_{1}^{2}+i M_{1} \Gamma_{1}}\left\langle N_{1} \mid \bar{l} \bar{\phi}\right\rangle .
$$

The residue yields the decay amplitude and, in particular, the $C P$ asymmetry. The RIS term can then be identified as the squared matrix element in the zero-width limit,

$$
\begin{aligned}
|\mathcal{M}(\bar{l} \bar{\phi} \rightarrow l \phi)|_{R I S}^{2} & =\lim _{\Gamma_{1} \rightarrow 0} 2 s^{2}\left(A_{11}-\sum_{k}\left(C_{1 k}+4 s D_{11 k}\right)\right) \\
& =16 \pi^{2} K_{11} M_{1}^{2}\left(1+2 \varepsilon_{1}^{M}+2 \varepsilon_{1}^{V}\right) \delta(x-1),
\end{aligned}
$$

where $\varepsilon_{1}^{M}$ and $\varepsilon_{1}^{V}$ are the familiar $C P$ asymmetries due to mixing and vertex correction, respectively $\left(M_{1} \ll M_{2}, M_{3}\right)$,

$$
\begin{aligned}
\varepsilon_{1}^{M} & =\frac{1}{8 \pi} \sum_{k} \frac{\operatorname{Im}\left\{K_{1 k}^{2}\right\}}{K_{11}} \frac{M_{1}}{M_{k}}, \\
\varepsilon_{1}^{V} & =-\frac{1}{8 \pi} \sum_{k} \frac{\operatorname{Im}\left\{K_{1 k}^{2}\right\}}{K_{11}} f\left(\frac{M_{k}^{2}}{M_{1}^{2}}\right) .
\end{aligned}
$$

In [4] it has been shown that the subtraction of the RIS term, which corresponds to the replacement $\left(1 / D_{1}^{2}+1 / R_{1}^{2}\right) \rightarrow\left(1 / D_{1}^{2}+1 / R_{1}^{2}\right)-(\pi / c) \delta(x-1)$, leads to Boltzmann equations with the expected properties, which have the equilibrium solution $N_{1}=N_{1}^{\mathrm{eq}}$, $N_{B-L}=0$.

It is now straightforward to write down the subtracted matrix element squared. Keeping only terms $\mathcal{O}\left(h^{4}\right)$, where the zero-width limit can be taken for $B_{1 i}$ and $\bar{E}_{11 k}$, one obtains the simple expressions $(i, j=2,3)$,

$$
\begin{aligned}
& |\mathcal{M}(\bar{l} \bar{\phi} \rightarrow l \phi)|_{\text {sub }}^{2}=\left|\mathcal{M}_{\Delta L=2}\right|_{+}^{2}+\left|\mathcal{M}_{\Delta L=2}\right|_{-}^{2}, \\
& |\mathcal{M}(l \phi \rightarrow \bar{l} \bar{\phi})|_{\text {sub }}^{2}=\left|\mathcal{M}_{\Delta L=2}\right|_{+}^{2}-\left|\mathcal{M}_{\Delta L=2}\right|_{-}^{2},
\end{aligned}
$$


with

$$
\begin{aligned}
\left|\mathcal{M}_{\Delta L=2}\right|_{+}^{2}= & 2 s^{2}\left\{\frac { K _ { 1 1 } ^ { 2 } } { M _ { 1 } ^ { 2 } } \left[\frac{1}{D_{1}^{2}}+\frac{1}{R_{1}^{2}}-\frac{\pi}{c} \delta(x-1)\right.\right. \\
& \left.+\frac{2}{x}-\frac{2}{x^{2}}\left(1+\frac{x+1}{D_{1}}\right) \ln (x+1)+\frac{2}{x D_{1}}\right] \\
& -6 \sum_{i} \operatorname{Re}\left(K_{1 i}^{2}\right) \frac{1}{M_{1} M_{i}}\left[\frac{1}{x}+\frac{1}{2 D_{1}}-\frac{(x+1)}{x^{2}} \ln (x+1)\right] \\
& \left.+3 \sum_{i, j} \operatorname{Re}\left(K_{i j}^{2}\right) \frac{1}{M_{i} M_{j}}\right\} \\
\left|\mathcal{M}_{\Delta L=2}\right|_{-}^{2}= & \frac{1}{2}\left(|\mathcal{M}(\bar{l} \bar{\phi} \rightarrow l \phi)|_{a v}^{2}-|\mathcal{M}(l \phi \rightarrow \bar{l} \bar{\phi})|_{a v}^{2}\right) \\
= & -32 \pi^{2} K_{11} M_{1}^{2}\left(\varepsilon_{1}^{M}+\varepsilon_{1}^{V}\right) \delta(x-1) .
\end{aligned}
$$

Note that the subtracted squared matrix element, contrary to the unsubtracted one, violates $C P$. To leading order in the coupling this part contributes only on-shell, and it is $\mathcal{O}\left(h^{2}\right)$ suppressed with respect to the leading Born term. Away from the pole, for $s \ll M_{1}^{2}$, one has

$$
\begin{aligned}
|\mathcal{M}(\bar{l} \bar{\phi} \rightarrow l \phi)|_{\text {sub }}^{2} & =|\mathcal{M}(l \phi \rightarrow \bar{l} \bar{\phi})|_{\text {sub }}^{2} \\
& =6 s^{2} \sum_{i j} \operatorname{Re}\left\{K_{i j}^{2}\right\} \frac{1}{M_{i} M_{j}} \\
& =\frac{6 s^{2}}{v^{4}} \operatorname{tr}\left(m_{\nu}^{\dagger} m_{\nu}\right)
\end{aligned}
$$

which is the crucial term leading to the upper bound on the neutrino masses [10].

We also have to take into account the $\Delta L=2$ process $l l \rightarrow \bar{\phi} \bar{\phi}$. The corresponding matrix elements reads

$$
\begin{aligned}
\left|\mathcal{M}_{\Delta L=2, t}\right|^{2}= & 2 s^{2}\left\{\frac{K_{11}^{2}}{M_{1}^{2}}\left[\frac{2}{x+1}+\frac{2}{x(x+2)} \ln (x+1)\right]\right. \\
& \left.+6 \sum_{i} \operatorname{Re}\left(K_{1 i}^{2}\right) \frac{1}{M_{1} M_{i}} \frac{1}{x} \ln (x+1)+3 \sum_{i, j} \operatorname{Re}\left(K_{i j}^{2}\right) \frac{1}{M_{i} M_{j}}\right\} .
\end{aligned}
$$

For small center of mass energies one again obtains

$$
\left|\mathcal{M}_{\Delta L=2, t}\right|^{2}=\frac{6 s^{2}}{v^{4}} \operatorname{tr}\left(m_{\nu}^{\dagger} m_{\nu}\right) .
$$


For the derivation of the upper bound on the light neutrino masses one needs the maximal $C P$ asymmetry for given $M_{1}, \widetilde{m}_{1}$ and $\bar{m}$. In this case also the complete $\Delta L=2$ matrix element depends just on these three variables. This is easily seen in the flavor basis where the Yukawa matrix $\tilde{h}$ connects light and heavy neutrino mass eigenstates. The matrix

$$
\Omega_{i j}=\frac{v}{\sqrt{m_{i} M_{j}}} \tilde{h}_{i j}
$$

is then orthogonal, $\Omega \Omega^{T}=\Omega^{T} \Omega=I$ [41], which implies

$$
\sum_{k} \tilde{h}_{i k} \tilde{h}_{j k} \frac{1}{M_{k}}=\frac{\sqrt{m_{i} m_{j}}}{v^{2}} \delta_{i j} .
$$

Using $\operatorname{Re}\left\{K_{1 i}^{2}\right\}=\operatorname{Re}\left\{K_{i 1}^{2}\right\}$, this implies for the interference term appearing in Eq. (190),

$$
\sum_{i \neq 1} \operatorname{Re}\left\{K_{1 i}^{2}\right\} \frac{1}{M_{i}}=-\frac{K_{11}^{2}}{M_{1}}+\sum_{j=1}^{3} \frac{m_{j}}{v^{2}} \operatorname{Re}\left\{\tilde{h}_{j 1}^{2}\right\} .
$$

The conditions

$$
\operatorname{Re}\left\{\tilde{h}_{21}^{2}\right\}=\operatorname{Re}\left\{\tilde{h}_{31}^{2}\right\}=0, \quad \operatorname{Re}\left\{\tilde{h}_{11}^{2}\right\}=\frac{m_{1} M_{1}}{v^{2}},
$$

yield a good approximation for the maximal $C P$ asymmetry [2]. The difference to the maximal $C P$ asymmetry [32] can then be treated as a perturbation, as discussed in sect. 4 . Eq. (198) then implies for the interference term

$$
\sum_{i \neq 1} \operatorname{Re}\left\{K_{1 i}^{2}\right\} \frac{1}{M_{i}}=-\frac{M_{1}}{v^{4}}\left(\widetilde{m}_{1}^{2}-m_{1}^{2}\right) .
$$

Inserting this expression in Eq. (190) one obtains for the $\Delta L=2$ matrix element in the case of maximal $C P$ asymmetry,

$$
\begin{aligned}
\left|\mathcal{M}_{\Delta L=2}\right|_{+}^{2}= & \frac{2 s^{2}}{v^{4}}\left\{\widetilde { m } _ { 1 } ^ { 2 } \left[\frac{1}{D_{1}^{2}}+\frac{1}{R_{1}^{2}}-\frac{\pi}{c} \delta(x-1)+\frac{2}{x}\left(1+\frac{1}{D_{1}}\right)-3\right.\right. \\
& \left.-\frac{2}{x^{2}}\left(1+\frac{x+1}{D_{1}}\right) \ln (x+1)\right] \\
& \left.+6\left(\widetilde{m}_{1}^{2}-m_{1}^{2}\right)\left[\frac{x+1}{x}+\frac{1}{2 D_{1}}-\frac{(x+1)}{x^{2}} \ln (x+1)\right]+3 \bar{m}^{2}\right\} .
\end{aligned}
$$

For the process $l l \rightarrow \bar{\phi} \bar{\phi}$ one obtains in the case of maximal $C P$ asymmetry,

$$
\begin{aligned}
\left|\mathcal{M}_{\Delta L=2, t}\right|^{2}= & \frac{2 s^{2}}{v^{4}}\left\{\widetilde{m}_{1}^{2}\left[\frac{2}{x+1}-3+\frac{2}{x(x+2)} \ln (x+1)\right]\right. \\
& \left.+6\left(\widetilde{m}_{1}^{2}-m_{1}^{2}\right)\left[1-\frac{1}{x} \ln (x+1)\right]+3 \bar{m}^{2}\right\} .
\end{aligned}
$$


For small energies, $s \ll M_{1}^{2}$, these matrix elements again reduce to

$$
\left|\mathcal{M}_{\Delta L=2}\right|_{+}^{2}=\left|\mathcal{M}_{\Delta L=2, t}\right|^{2}=\frac{6 s^{2}}{v^{4}} \bar{m}^{2},
$$

whereas for intermediate energies $M_{1}^{2} \ll s \ll M_{2,3}^{2}$ one finds

$$
\left|\mathcal{M}_{\Delta L=2}\right|_{+}^{2}=\left|\mathcal{M}_{\Delta L=2, t}\right|^{2}=\frac{6 s^{2}}{v^{4}}\left(\widetilde{m}_{1}^{2}+\bar{m}^{2}-2 m_{1}^{2}\right)
$$

Following $[4,5]$, it has been standard practice [6]-[11] to determine $|\mathcal{M}|_{\text {sub }}^{2}$ by computing the Born diagrams for the $2 \rightarrow 2$ process with Breit-Wigner propagator and dropping $1 / R_{1}$, the imaginary part of the propagator, since in the zero width limit

$$
\begin{aligned}
\frac{1}{s-M_{1}^{2}+i M_{1} \Gamma_{1}} & =\frac{1}{M_{1}^{2}}\left(\frac{1}{D_{1}(x)}-i \frac{1}{R_{1}(x)}\right) \\
& \stackrel{\Gamma_{1} \rightarrow 0}{\longrightarrow}-i \pi \delta\left(s-M_{1}^{2}\right) .
\end{aligned}
$$

Recently, it has been pointed out that this procedure is not correct [12]. In a toy model, the same conclusion has been reached in [42]. Indeed, the described procedure leads to a subtracted squared matrix element which contains terms $\propto 1 / D_{1}^{2}[5]$, implying $|\mathcal{M}|_{\text {sub }}^{2}=$ $\mathcal{O}\left(h^{2}\right)$ on-shell, in contradiction with Eq. (190). The zero-width limits of the squared amplitude and the squared imaginary part are different. This was overlooked in the past, leading to an overestimate of the washout rate due to inverse decays by $50 \%$ [12]. The RIS term has to be subtracted from the full $2 \rightarrow 2$ cross section, not just from the Born cross section, in order to obtain the crucial $C P$ violating contribution proportional to $\varepsilon_{1}{ }^{5}$.

Let us now consider the Boltzmann equation for the density of lepton doublets, assuming kinetic equilibrium,

$$
\begin{aligned}
\frac{d n_{l}}{d t}+3 H n_{l}= & \frac{n_{N_{1}}}{n_{N_{1}}^{\mathrm{eq}}} \gamma^{\mathrm{eq}}\left(N_{1} \rightarrow l \phi\right)-\frac{n_{l}}{n_{l}^{\mathrm{eq}}} \gamma^{\mathrm{eq}}\left(l \phi \rightarrow N_{1}\right) \\
& +\frac{n_{\bar{l}}}{n_{l}^{\mathrm{eq}}} \gamma_{\mathrm{sub}}^{\mathrm{eq}}(\bar{l} \bar{\phi} \rightarrow l \phi)-\frac{n_{l}}{n_{l}^{\mathrm{eq}}} \gamma_{\mathrm{sub}}^{\mathrm{eq}}(l \phi \rightarrow \bar{l} \bar{\phi}) \\
& +\gamma^{\mathrm{eq}}(\bar{\phi} \bar{\phi} \rightarrow l l)-\left(\frac{n_{l}}{n_{l}^{\mathrm{eq}}}\right)^{2} \gamma^{\mathrm{eq}}(l l \rightarrow \bar{\phi} \bar{\phi}) .
\end{aligned}
$$

\footnotetext{
${ }^{5}$ Note that in Ref. [12] $\gamma_{N_{s}}^{\text {on-shell }}(L H \rightarrow \bar{L} \bar{H})$ is $C P$ violating, using a given $C P$ asymmetry $\epsilon_{N_{1}}$ not determined by the $2 \rightarrow 2$ processes. It is different from the on-shell part of $\gamma_{N_{s}}(L H \rightarrow \bar{L} \bar{H})$ which, as a tree-level rate, conserves $C P$. As discussed above, the correct subtraction term is obtained from the full reaction rate including vertex and self-energy corrections to $\mathcal{O}\left(h^{4}\right)$ by separating the on-shell part from the interference terms. This procedure automatically yields the correct $C P$ asymmetry $\epsilon_{N_{1}}$.
} 
Here, $\gamma^{\text {eq }}$ are the usual reaction densities in thermal equilibrium and we have assumed that the Higgs doublets $\phi$ are in thermal equilibrium, neglecting their chemical potential. The $C P$ asymmetry $\varepsilon_{1}$ is defined in such a way that

$$
\begin{aligned}
\gamma^{\mathrm{eq}}\left(N_{1} \rightarrow l \phi\right) & =\gamma^{\mathrm{eq}}\left(\bar{l} \bar{\phi} \rightarrow N_{1}\right)=\frac{1+\varepsilon_{1}}{2} \gamma_{N_{1}}, \\
\gamma^{\mathrm{eq}}\left(N_{1} \rightarrow \bar{l} \bar{\phi}\right) & =\gamma^{\mathrm{eq}}\left(l \phi \rightarrow N_{1}\right)=\frac{1-\varepsilon_{1}}{2} \gamma_{N_{1}} .
\end{aligned}
$$

Further, for the $2 \rightarrow 2$ processes we have

$$
\begin{aligned}
\gamma_{\mathrm{sub}}^{\mathrm{eq}}(\bar{l} \bar{\phi} \rightarrow l \phi) & =\gamma_{\Delta L=2,+}^{\mathrm{eq}}-\frac{1}{2} \varepsilon_{1} \gamma_{N_{1}}, \\
\gamma_{\mathrm{sub}}^{\mathrm{eq}}(l \phi \rightarrow \bar{l} \bar{\phi}) & =\gamma_{\Delta L=2,+}^{\mathrm{eq}}+\frac{1}{2} \varepsilon_{1} \gamma_{N_{1}}, \\
\gamma^{\mathrm{eq}}(\bar{\phi} \bar{\phi} \leftrightarrow l l) & =\gamma^{\mathrm{eq}}(\overline{l l} \leftrightarrow \phi \phi)=\gamma_{\Delta L=2, t}^{\mathrm{eq}} .
\end{aligned}
$$

Introducing a lepton, or $B-L$ asymmetry,

$$
n_{l}=n_{l}^{\mathrm{eq}}-\frac{1}{2} n_{B-L}, \quad n_{\bar{l}}=n_{l}^{\mathrm{eq}}+\frac{1}{2} n_{B-L}
$$

assuming $n_{B-L}=n_{\bar{l}}-n_{l}=\mathcal{O}\left(\varepsilon_{1}\right)$, and keeping only terms $\mathcal{O}\left(\varepsilon_{1}\right)$, one obtains the kinetic equation for the $B-L$ asymmetry

$$
\frac{d n_{B-L}}{d t}+3 H n_{B-L}=-\varepsilon_{1}\left(\frac{n_{N_{1}}}{n_{N_{1}}^{\mathrm{eq}}}-1\right) \gamma_{N_{1}}-\frac{n_{B-L}}{n_{l}^{\mathrm{eq}}}\left(\frac{1}{2} \gamma_{N_{1}}+\gamma_{\Delta L=2}\right)
$$

where

$$
\gamma_{\Delta L=2}=2 \gamma_{\Delta L=2,+}^{\mathrm{eq}}+2 \gamma_{\Delta L=2, t}^{\mathrm{eq}}
$$

The $C P$ violating part of $\gamma_{\text {sub }}^{\text {eq }}$ yields the term $+\varepsilon_{1} \gamma_{N_{1}}$ which guarantees that for $n_{N_{1}}=n_{N_{1}}^{\text {eq }}$ no asymmetry is generated. Note that the old procedure for subtracting the RIS part of the $2 \rightarrow 2$ process would have led to a contribution $\frac{3}{4} \gamma_{N_{1}}$ in the washout term rather than $\frac{1}{2} \gamma_{N_{1}}$ [12]. Neglecting the off-shell contribution $\gamma_{\Delta L=2}$, using the relation

$$
\gamma_{N_{1}}=n_{N_{1}}^{\mathrm{eq}} z H D=2 n_{l}^{\mathrm{eq}} z H W_{I D}
$$

and changing variables from $t$ to $z=M_{1} / T$ and from number densities to particle numbers in a comoving volume (cf. [10]), one obtains the Boltzmann equation (13).

In order to obtain the kinetic equation (212) the correct identification of the RIS term is essential. It is therefore of crucial importance to derive this equation from first principles. In the case of non-relativistic heavy neutrinos, i.e. $T<M_{1}$, this has been done 
in [38]. Note that in the strong washout regime, where $T_{B}<M_{1}$, the decaying neutrinos are indeed non-relativistic. Eq. (49) of [38] gives the analogue of (212) for Boltzmann distribution functions rather than the integrated number densities. The starting point of this derivation are the Kadanoff-Baym equations which describe the full quantum mechanical problem. Leptogenesis is then studied as a process close to thermal equilibrium. As a consequence, the deviations of distribution functions from equilibrium distribution functions, $\delta f_{N}(t, p)$ and $\delta f_{l}(t, k)$ appear from the beginning ${ }^{6}$. For simplicity, in Eq. (49) of ref. [38] the contribution to the washout term from interferences with the heavy neutrinos $N_{2}$ and $N_{3}$ has been neglected. Otherwise the result is identical to Eq. (212). In particular, the relative size of the driving term for the asymmetry, which is proportional to $\varepsilon_{1}$, and the washout term due to inverse decays agrees with (212). It is important to derive the Boltzmann equations and the reaction densities within a full quantum mechanical treatment also for relativistic heavy neutrinos, in particular in the resonance region $T \sim M_{1}$.

It is instructive to discuss the different contributions to $\gamma_{\Delta L=2}$, the reaction density corresponding to the averaged matrix element squared

$$
\begin{aligned}
\left|\mathcal{M}_{\Delta L=2}\right|^{2}= & 2\left|\mathcal{M}_{\Delta L=2}\right|_{+}^{2}+2\left|\mathcal{M}_{\Delta L=2, t}\right|^{2} \\
= & \frac{4 s^{2}}{v^{4}}\left\{\widetilde{m}_{1}^{2}\left(\frac{1}{D_{1}^{2}}+\frac{1}{R_{1}^{2}}\right)-32 \pi^{2} \frac{\widetilde{m}_{1} v^{2}}{M_{1}} \frac{1}{x^{2}} \delta(x-1)+24 \bar{m}^{2}\right. \\
& +\tilde{m}_{1}^{2}\left[6+\frac{8}{x}+\frac{2}{x+1}+\frac{1}{D_{1}}\left(3+\frac{2}{x}\right)\right. \\
& \left.-\frac{2}{x^{2}}\left(6 x+3+\frac{2}{x+2}+\frac{x+1}{D_{1}}\right) \ln (x+1)\right] \\
& \left.-6 m_{1}^{2}\left[2+\frac{1}{x}+\frac{1}{2 D_{1}}-\frac{(2 x+1)}{x^{2}} \ln (x+1)\right]\right\}
\end{aligned}
$$

where we have again assumed the relation (199). The different contributions to the washout term $W$ are shown in Fig. 13. The term proportional to $\bar{m}^{2}$, as well as the contributions from the last three lines at high temperatures, $M_{1} \ll T \ll M_{2,3}$, give a simple power law behavior, corresponding to Eqs. (202) and (203). At low temperatures, the term proportional to $m_{1}^{2}$ rapidly approaches zero and becomes negligible.

It can be seen clearly that for $z \lesssim 30$ the contributions from the first two terms in Eq. (215) cancel each other to a very good approximation, corresponding to the subtrac-

\footnotetext{
${ }^{6}$ The part of the Lagrangian involving left- and right-handed neutrinos has a U(1) symmetry which implies $\delta f_{l}(t, k)=-\delta f_{\phi}(t, k)$. Here we have assumed that due to the other interactions in the standard model $\delta f_{\phi}(t, k)=0$.
} 


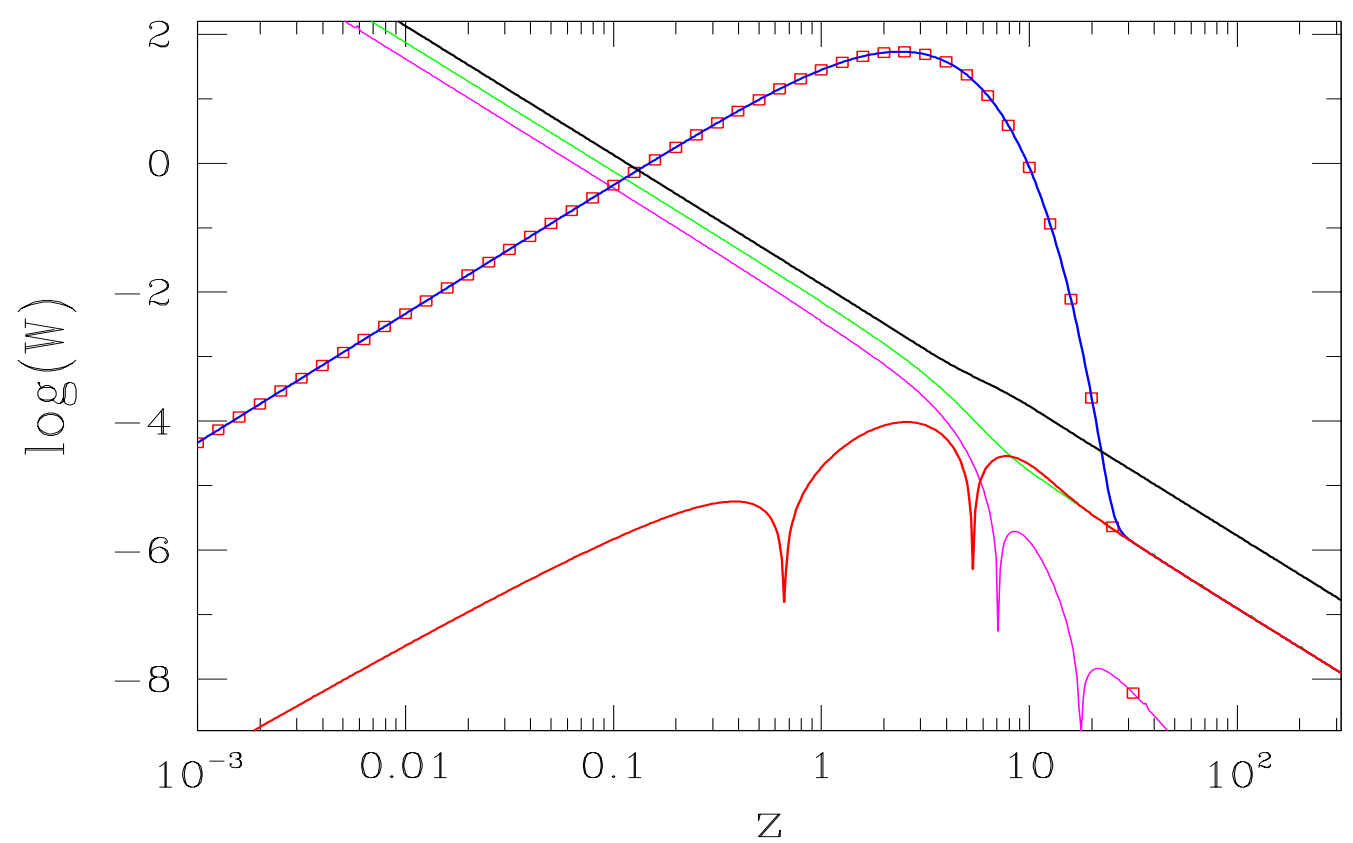

Figure 13: Absolute values of the different contributions to the washout term $W$ from the averaged squared matrix element in eq. (215) for $M_{1}=10^{10} \mathrm{GeV}, \bar{m}=0.05 \mathrm{eV}$, $m_{1}=\bar{m} / \sqrt{3}$ and $\widetilde{m}_{1}=0.03 \mathrm{eV}$. The solid line is the term proportional to $1 / D_{1}^{2}+1 / R_{1}^{2}$, the black squares the contribution from the delta function, the dotted line the term proportional to $\widetilde{m}_{1}^{2}$ in the second and third lines of eq. (215), the dashed line the term proportional to $m_{1}^{2}$, and the dashed-dotted line is the term proportional to $\bar{m}^{2}$.

tion of RIS contributions. However, the term proportional to $1 / D_{1}^{2}+1 / R_{1}^{2}$ has a different low temperature limit than the delta function and cancels against the term in the second and third lines of Eq. (215) for $z \gtrsim 30$.

Finally, this discussion is only applicable if off-shell and RIS contributions can be separated. This is related to the usual approximation that the right handed neutrinos can be considered as asymptotic free states, i.e. that one can write down a Boltzmann equation for them, which is the case if their width is small, i.e.,

$$
c=\frac{\Gamma_{1}}{M_{1}}<\delta,
$$

where $\delta$ is some constant smaller than one. This translates into the following condition for $\widetilde{m}_{1}$ :

$$
\widetilde{m}_{1}<\delta 0.76 \mathrm{eV}\left(\frac{10^{15} \mathrm{GeV}}{M_{1}}\right)
$$


We have checked numerically that the separation of on-shell and off-shell contributions works well, as long as Eq. (217) with $\delta=0.1$ is fulfilled.

\section{Appendix B}

The scattering rates are expressed through the reaction densities rates and these, in turn, through the reduced cross sections,

$$
\Gamma_{\phi, t(s)}^{\left(N_{1}\right)}=\frac{\gamma_{\phi, t(s)}}{n_{N_{1}}^{\mathrm{eq}}}=\frac{M_{1}}{32 g_{N_{1}} \pi^{2}} \frac{\mathcal{I}_{\phi, t(s)}(z)}{K_{2}(z) z^{3}},
$$

where we introduced the following integrals

$$
\mathcal{I}_{\phi, t(s)}(z)=\int_{z^{2}}^{\infty} d \psi \hat{\sigma}_{\phi, t(s)}(\psi) \sqrt{\psi} K_{1}(\sqrt{\psi}) .
$$

The reduced cross sections can be written in the following form [8]:

$$
\hat{\sigma}_{\phi, t(s)}=\frac{3 \alpha_{\mu}}{4 \pi} \frac{M_{1} \tilde{m}_{1}}{v^{2}} f_{\phi, t(s)}(x)
$$

with $x \equiv \psi / z^{2}, \alpha_{\mu}=m_{t}^{2} / v^{2}$ and where we defined the following functions:

$$
\begin{gathered}
f_{\phi, t}(x)=\frac{x-1}{x}\left[\frac{x-2+2 a_{h}}{x-1+a_{h}}+\frac{1-2 a_{h}}{x-1} \ln \left(\frac{x-1+a_{h}}{a_{h}}\right)\right], \\
f_{\phi, s}(x)=\left(\frac{x-1}{x}\right)^{2} .
\end{gathered}
$$

with $a_{h}=\left(M_{\mathrm{h}} / M_{1}\right)^{2}$. The functions $f_{\phi, t(s)}(z)$ are then defined as:

$$
f_{\phi, t(s)}(z)=\frac{\int_{z^{2}}^{\infty} d \psi f_{\phi, t(s)}\left(\psi / z^{2}\right) \sqrt{\psi} K_{1}(\sqrt{\psi})}{z^{2} K_{2}(z)},
$$

and in this way the Eq. (73) for $S_{t}$ follows.

Similarly to the Eq. (25) for $K_{2}(z)$, the modified Bessel function $K_{3}(z)$ can be approximated by the analytical expression

$$
K_{3}(z) \simeq \frac{1}{z^{3}} \sqrt{1+\frac{\pi}{2} z} e^{-z}\left(\frac{945}{128}+\frac{35}{8} z+z^{2}\right) .
$$

For $M_{h} / M_{1}=10^{-5}$ and small $K, z_{\text {eq }}$ is well described by

$$
z_{\text {eq }}=0.4+1.3 \ln \left(1+K^{-0.88}\right) \text {. }
$$




\section{References}

[1] M. Fukugita, T. Yanagida, Phys. Lett. B 174 (1986) 45

[2] W. Buchmüller, P. Di Bari, M. Plümacher, Nucl. Phys. B 665 (2003) 445

[3] E. W. Kolb, M. S. Turner, The Early Universe, Addison-Wesley, New York, 1990

[4] E. W. Kolb, S. Wolfram, Nucl. Phys. B 172 (1980) 224; Nucl. Phys. B 195 (1982) $542(\mathrm{E})$

[5] J. A. Harvey, E. W. Kolb, D. B. Reiss, S. Wolfram, Nucl. Phys. B 201 (1982) 16

[6] M. A. Luty, Phys. Rev. D 45 (1992) 455

[7] M. Plümacher, Z. Phys. C 74 (1997) 549

[8] M. Plümacher, Nucl. Phys. B 530 (1998) 207

[9] R. Barbieri, P. Creminelli, A. Strumia, N. Tetradis, Nucl. Phys. B 575 (2000) 61

[10] W. Buchmüller, P. Di Bari, M. Plümacher, Nucl. Phys. B 643 (2002) 367

[11] A. Pilaftsis and T. E. J. Underwood, hep-ph/0309342

[12] G. F. Giudice, A. Notari, M. Raidal, A. Riotto, A. Strumia, hep-ph/0310123

[13] S. Yu. Khlebnikov, M. E. Shaposhnikov, Nucl. Phys. B 308 (1988) 885;

J. A. Harvey, M. S. Turner, Phys. Rev. D 42 (1990) 3344

[14] L. Covi, N. Rius, E. Roulet, F. Vissani, Phys. Rev. D 57 (1998) 93

[15] J. N. Fry, M. S. Turner, Phys. Rev. D 24 (1981) 3341

[16] R. M. Corless et al., Adv. Comp. Math., Vol. 5 (1996) 329

[17] F. Chapeau-Blondeau and A. Monir, IEEE Trans. Signal Processing, Vol. 50 (2002), 2160

[18] P. Di Bari, AIP Conf. Proc. 655 (2003) 208 [hep-ph/0211175]

[19] M. Fujii, K. Hamaguchi, T. Yanagida, Phys. Rev. D 65 (2002) 115012

[20] WMAP Collaboration, D. N. Spergel et al., Astrophys. J. Suppl. 148 (2003) 175. 
[21] Max Tegmark et al., astro-ph/0310723.

[22] K. Hamaguchi, H. Murayama, T. Yanagida, Phys. Rev. D 65 (2002) 043512

[23] For recent discussions and references, see

Z. Z. Xing, hep-ph/0307359;

G. C. Branco, hep-ph/0309215;

W. Rodejohann, hep-ph/0311142;

S. Davidson, R. Kitano, hep-ph/0312007

[24] S. Davidson, A. Ibarra, Phys. Lett. B 535 (2002) 25

[25] G. L. Fogli, E. Lisi, A. Marrone, D. Montanino, A. Palazzo, A. M. Rotunno, hepph/0310012.

[26] M. H. Ahn et al., K2K Collaboration, Phys. Rev. Lett. 90 (2003) 041801;

M. Shiozawa et al., SK Collaboration in Neutrino 2002, Proc. to appear.

[27] Q.R. Ahmad et al, SNO Collaboration, nucl-ex/0309004;

K. Eguchi et al., KamLAND Collaboration, Phys. Rev. Lett. 90 (2003) 021802

[28] P. H. Chankowski, K. Turzyński, Phys. Lett. B 570 (2003) 198

[29] M. Flanz, E. A. Paschos, U. Sarkar, Phys. Lett. B 345 (1995) 248; Phys. Lett. B 384 (1996) $487(\mathrm{E})$

[30] L. Covi, E. Roulet, F. Vissani, Phys. Lett. B 384 (1996) 169

[31] G. F. Giudice, E. W. Kolb and A. Riotto, Phys. Rev. D 64 (2001) 023508

[32] T. Hambye, Y. Lin, A. Notari, M. Papucci, A. Strumia, hep-ph/0312203

[33] W. Buchmüller, P. Di Bari, M. Plümacher, Phys. Lett. B 547 (2002) 128

[34] S. Antusch, J. Kersten, M. Lindner, M. Ratz, Nucl. Phys. B 674 (2003) 401

[35] W. Buchmüller, M. Plümacher, Phys. Lett. B 511 (2001) 74

[36] W. Buchmüller, M. Plümacher, Int. J. Mod. Phys. A 15 (2000) 5047

[37] G. Lazarides, Q. Shafi, Phys. Lett. B 258 (1991) 305;

H. Murayama, T. Yanagida, Phys. Lett. B 322 (1994) 349

[38] W. Buchmüller, S. Fredenhagen, Phys. Lett. B 483 (2000) 217 
[39] W. Buchmüller, M. Plümacher, Phys. Lett. B 431 (1998) 354

[40] E. Roulet, L. Covi, F. Vissani, Phys. Lett. B 424 (1998) 101

[41] J. A. Casas, A. Ibarra, Nucl. Phys. B 618 (2001) 171

[42] R. F. Sawyer, hep-ph/0312158 